The Annals of Applied Probability

2014, Vol. 24, No. 2, 811-856

DOI: $10.1214 / 13$-AAP936

(C) Institute of Mathematical Statistics, 2014

\title{
TIME-CHANGED CIR DEFAULT INTENSITIES WITH TWO-SIDED MEAN-REVERTING JUMPS
}

\author{
By Rafael Mendoza-Arriaga and Vadim Linetsky ${ }^{1}$ \\ University of Texas at Austin and Northwestern University
}

\begin{abstract}
The present paper introduces a jump-diffusion extension of the classical diffusion default intensity model by means of subordination in the sense of Bochner. We start from the bi-variate process $(X, D)$ of a diffusion state variable $X$ driving default intensity and a default indicator process $D$ and time change it with a Lévy subordinator $\mathcal{T}$. We characterize the time-changed process $\left(X_{t}^{\phi}, D_{t}^{\phi}\right)=\left(X\left(\mathcal{T}_{t}\right), D\left(\mathcal{T}_{t}\right)\right)$ as a Markovian-Itô semimartingale and show from the Doob-Meyer decomposition of $D^{\phi}$ that the default time in the time-changed model has a jump-diffusion or a pure jump intensity. When $X$ is a CIR diffusion with mean-reverting drift, the default intensity of the subordinate model $(S u b C I R)$ is a jump-diffusion or a pure jump process with mean-reverting jumps in both directions that stays nonnegative. The SubCIR default intensity model is analytically tractable by means of explicitly computed eigenfunction expansions of relevant semigroups, yielding closed-form pricing of credit-sensitive securities.
\end{abstract}

1. Introduction. The classical Cox, Ingersoll and Ross (1985) (CIR)/ Feller (1951) square-root diffusion has been a workhorse in the stochastic intensity approach to the modeling of default risk in financial markets since the seminal work of Jarrow, Lando and Turnbull (1997) and Duffie and Singleton (1999) on reduced-form default modeling; see monographs Bielecki and Rutkowski (2004), Duffie and Singleton (2003) and Jeanblanc, Yor and Chesney (2009) for surveys. In this framework, the default time can be thought of as the first jump time of a doubly stochastic Poisson process (Cox process) with stochastic intensity following a diffusion process. The attractiveness of the CIR diffusion as the model for intensity stems from, on

Received September 2012; revised March 2013.

${ }^{1}$ Supported by NSF Grants DMS-08-02720 and CMMI-1030486.

AMS 2000 subject classifications. Primary 91G40; secondary 60J75, 91G30, 60G55.

Key words and phrases. Default, default intensity, credit spread, corporate bond, credit derivative, CIR process, time change, subordinator, Bochner subordination, jump-diffusion process, state dependent Lévy measure, spectral expansion.

This is an electronic reprint of the original article published by the Institute of Mathematical Statistics in The Annals of Applied Probability, 2014, Vol. 24, No. 2, 811-856. This reprint differs from the original in pagination and typographic detail. 
one hand, its dynamics and, on the other hand, its analytical tractability. If the coefficient of the linear term in the drift is negative, and the constant term is positive, then CIR diffusion is mean-reverting, which is an important empirical feature observed in credit markets. At the same time, the process stays nonnegative due to vanishing volatility and positive drift near the origin. Its analytical tractability stems, on one hand, from its close connection with Bessel processes [Pitman and Yor (1982), Göing-Jaeschke and Yor (2003), Chapter 6 of Jeanblanc, Yor and Chesney (2009), Revuz and Yor (1999)] and, on the other hand, from its membership in the class of affine processes [Duffie and Kan (1996), Duffie, Pan and Singleton (2000), Duffie, Filipović and Schachermayer (2003), Keller-Ressel, Schachermayer and Teichmann (2011)]. The former connection yields explicit expressions for the CIR transition density and the associated Feynman-Kac semigroup, while the later connection yields an explicit expression for Laplace transform of the time integral of the CIR process, giving rise to a closed-form solution for the survival probability in the CIR default intensity model that essentially coincides with the expression for the bond price in CIR interest rate model. These properties lead to analytical pricing for a wide range of credit-sensitive instruments in CIR-based models [e.g., Brigo and Alfonsi (2005), Bielecki, Jeanblanc and Rutkowski (2011)].

A limitation of the CIR default intensity model is its inability to capture jumps in credit spreads and prices of credit-sensitive securities (other than the default event itself). This led a number of authors to introduce jumps into the CIR model [Duffie and Garleanu (2001), Filipović (2001), Brigo and El-Bachir (2006, 2010)]. To preserve analytical tractability, all of the models considered in the literature so far have been in the affine class. The most general extensions of the one-dimensional CIR diffusion with jumps that remain in the affine class are continuous state branching processes with immigration (CBI) of Kawazu and Watanabe (1971); see Filipović (2001) for a detailed treatment in the context of applications to interest rate term structure modeling. Roughly speaking, CBI-processes are nonnegative Feller processes with CIR-type diffusion components and one-sided, positive jumps with the compensator measure of the form $m(d y)+x \mu(d y)$, where $m$ is the Lévy measure of a subordinator, and $\mu(d y)$ is the Lévy measure of a spectrally positive Lévy process; see Theorem 4.3 in Filipović (2001) for the explicit expression of their infinitesimal generator and the summary of their properties.

A limitation of CBI-processes is the one-sided nature of their jumps. From the standpoint of financial applications, their sample path behavior is somewhat unnatural. CBI processes can only jump up, and can never jump down. Assuming the drift of the CBI-process is mean-reverting, if the process experiences a large jump up bringing it far away from its long-run mean, the only mechanism for it to return back to its long-run mean is via its 
continuous mean-reverting drift, with no possibility to jump back down. Moreover, jumps of CBI-processes are either state independent (governed by $m$ if $\mu=0$ ), or depend linearly of the current state via $x$ multiplying $\mu$. The one-sided nature of jumps and their affine dependence on the state are common to general affine processes, for example, Cuchiero et al. (2011a) and Cuchiero et al. (2011b). However, this is in contrast to the behavior often observed in financial markets where a jump in one direction may be followed by a jump in the opposite direction. This behavior is often observed in energy markets, where mean-reverting models are commonly used to capture the spike-like behavior of the spot price of electricity [e.g., Barlow (2002), Geman and Roncoroni (2006) and Meyer-Brandis and Tankov (2008)]. This is also relevant in credit markets, where the succession of good and bad news about the financial health of an obligor, such as a firm or a sovereign viewed by the markets to be in distress, can result in sharp changes in its market credit spreads over relatively short periods of time (witness the recent behavior of some European credit spreads sea-sawing under the influence of the rapidly changing flow of economic and political news). Recent empirical literature studying positive and negative jumps in credit spreads includes Zhang, Zhou and Zhu (2009), Elkamhi et al. (2012) and Kita (2012).

This paper proposes a new approach to introducing more realistic twosided jump behavior into diffusion intensity models via Bochner's subordination. We start with a nonnegative diffusion intensity model and time change it with a subordinator, that is, a nonnegative Lévy process with positive jumps and nonnegative drift. We show that this results in a jump-diffusion (when the subordinator has a positive drift) or a pure-jump (when the subordinator is driftless) intensity model with two-sided jumps that stays nonnegative. In particular, when the diffusion is CIR, the time-changed model possesses a nonnegative intensity process with two-sided, mean-reverting jumps. The compensator measure of this intensity process is state-dependent, and the state-dependence is such that it automatically prevents the process from going negative. While the process can experience downward jumps, the magnitude of negative jumps depends on the pre-jump state of the process to keep the process nonnegative. While the structure of the process is highly state-dependent (and obviously nonaffine), remarkably, the model remains fully analytically tractable by means of eigenfunction expansions.

The rest of the paper is organized as follows. In Section 2 we review diffusion intensity models in the particular setting convenient for our purposes. Namely, we consider a bi-variate process $(X, D)$, where $X$ is the state variable following a nonnegative diffusion process (pre-intensity), and $D$ is an event indicator process $D$. The bi-variate process is a Markov process on $\mathbb{R}_{+} \cup\{0,1\}$ and a semimartingale. This section contains a detailed discussion of the bi-variate process $(X, D)$ both from the Markovian and from the semimartingale points of view. While the diffusion intensity model is very 
well known, this detailed presentation in the bi-variate form is provided for the reader's convenience to set up notation in preparation for our treatment of the time-changed (subordinated) model by both Markovian and semimartingale methods. We note that this bi-variate point of view of diffusion default intensity models is also followed in some interesting recent papers by Bielecki et al. (2008), Bielecki et al. (2012) and Bielecki et al. (2013) in the context of pricing multi-name credit derivatives. In Section 3 we time change the bi-variate process $(X, D)$ with a Lévy subordinator $\mathcal{T}$ with Laplace exponent $\phi$. The resulting time-changed process $\left(X^{\phi}, D^{\phi}\right)$ is a Markov process with its infinitesimal generator given by the Phillips theorem. We explicitly compute the generator from the Phillips theorem and obtain its representation as an integro-differential operator. Being a time change of a semimartingale, the bi-variate process is also a semimartingale. We then identify its predictable characteristics from the generator and obtain Lévy-Itô decomposition of $X^{\phi}$, Doob-Meyer decomposition of $D^{\phi}$ and Itô formula for functions $f\left(t, X^{\phi}, D^{\phi}\right)$. We then identify the process $D^{\phi}$ with the default indicator in our model, so that the default time is the jump time of $D^{\phi}$, and from its Doob-Meyer decomposition identify explicitly the default intensity as $\lambda_{t}^{\phi}=\left(1-D_{t}^{\phi}\right) k^{\phi}\left(X_{t}^{\phi}\right)$, where $k^{\phi}(x)$ is an explicitly determined positive function. In Section 4 we apply the results to the pricing of credit-sensitive securities. In Section 5 we detail the eigenfunction expansion approach to calculate the semigroup associated to the bi-variate process $\left(X^{\phi}, D^{\phi}\right)$ and, in particular, to calculate the survival probability and prices of defaultable securities. In Section 6 we specialize $X$ to be the CIR diffusion and thus obtain the subordinate CIR (SubCIR) default intensity model. This section contains explicit expressions of all the quantities relevant to the SubCIR model and, in particular, explicit eigenfunction expansions for the SubCIR semigroups. These explicit solutions are then applied to give numerical illustrations of the SubCIR default intensity model.

2. The diffusion default intensity model. We start with a complete probability space $(\Omega, \mathcal{F}, \mathbb{P})$ on which a one-dimensional standard Brownian motion $\left\{B_{t}, t \geq 0\right\}$ is defined. Let $\mathbb{F}^{B}=\left(\mathcal{F}_{t}^{B}\right)_{t \geq 0}$ denote its completed natural filtration. We model the state variable as the unique strong solution of the stochastic differential equation (SDE)

$$
X_{t}=x+\int_{0}^{t} b\left(X_{u}\right) d u+\int_{0}^{t} \sigma\left(X_{u}\right) d B_{u}, \quad t \geq 0 .
$$

We assume that the drift and diffusion coefficients $b(x)$ and $\sigma(x)$ are continuous on $(0, \infty), \sigma(x)>0$ on $(0, \infty)$, and are such that for each positive initial condition $X_{0}=x>0$ this SDE admits a unique strong solution that stays nonnegative for all $t>0$. Thus the state variable $\left(X_{t}\right)_{t \geq 0}$ is a onedimensional diffusion, as well as a nonnegative continuous semimartingale. 
Under our assumptions, the boundary at zero is either natural (in which case the process cannot reach zero when started from a positive value $x>0$, and cannot be started at zero), entrance [in which case the process cannot reach zero when started from a positive value $x>0$, but can be started at zero, in which case it instantaneously enters the interval $(0, \infty)$ and never comes back to zero], instantaneously reflecting, or absorbing (in which case $X_{t}=0$ for all $t \geq T_{0}$, where $T_{0}$ is the first hitting time of zero). We refer the reader to Ethier and Kurtz (1986), pages 366-367, and Borodin and Salminen (2002), Chapter II, for detailed expositions of Feller's classification of boundaries of one-dimensional diffusions. In this paper we exclude absorption, assuming that zero is either unattainable (natural or entrance), or an instantaneously reflecting boundary. Since we assume that the SDE (2.1) has a unique strong solution, the process does not explode to infinity when started from any $x>0$; that is, infinity is an unattainable boundary. The state space of the diffusion $X$ will be denoted by $I$, and $I=(0, \infty)$ if 0 is unattainable (natural or entrance) or $I=[0, \infty)$ if zero is instantaneously reflecting.

ExAmple 2.1 (The CIR SDE). The key example of interest to us in this paper is the CIR SDE with

$$
\sigma(x)=\sigma \sqrt{x} \quad \text { with } \sigma>0, \quad b(x)=\kappa(\theta-x) \quad \text { with } \kappa \theta>0 .
$$

Surveys of CIR processes and their relationship with Bessel processes can be found in Göing-Jaeschke and Yor (2003) and Jeanblanc, Yor and Chesney (2009), Section 6.3. The drift coefficient $b(x)$ is Lipschitz, and the diffusion coefficient $\sigma(x)$ satisfies the Yamada-Watanabe condition [cf. Revuz and Yor (1999), Theorem IX.1.7], so the SDE has a unique strong solution for any $x \geq 0$. Since for $\theta=0$ and $x=0$ the solution is $X_{t}=0$, by the comparison theorem for one-dimensional SDEs [cf. Revuz and Yor (1999), Theorem IX.3.7], the solutions for $\kappa \theta>0$ and $x \geq 0$ stay nonnegative, $X_{t} \geq 0$ for all $t \geq 0$. Furthermore, when the Feller condition is satisfied, $2 \kappa \theta \geq \sigma^{2}$, the process stays strictly positive when started from any $x>0$, that is, $\mathbb{P}\left(T_{0}=\infty\right)=1$, where $T_{0}$ is the first hitting time of zero. It can also be started from $x=0$, in which case it immediately enters the interval $(0, \infty)$ and stays strictly positive for all $t>0$. In this case the boundary at zero is an entrance boundary. When the Feller condition is not satisfied, $0<2 \kappa \theta<\sigma^{2}$, the process can reach zero when started from $x>0$, and zero is an instantaneously reflecting boundary.

Let $C([0, \infty])$ denote the Banach space of functions continuous on $(0, \infty)$ and such that the limits $\lim _{x \rightarrow 0} f(x)$ and $\lim _{x \rightarrow \infty} f(x)$ exist and are finite and endowed with the usual supremum norm. As shown in, for example, Ethier and Kurtz (1986), page 366, the transition function $P_{t}^{0}(x, d y)=$ $\mathbb{P}\left(X_{t}^{x} \in d y\right)$ of the diffusion process $X^{x}$ started at $x$ defines a Feller semi- 
group $\left(\mathcal{P}_{t}^{0}\right)_{t \geq 0}$ acting on $C([0, \infty])$ by

$$
\mathcal{P}_{t}^{0} f(x)=\mathbb{E}^{x}\left[f\left(X_{t}\right)\right]=\int_{I} f(y) P_{t}^{0}(x, d y),
$$

where $\mathbb{E}^{x}$ denotes the expectation corresponding to the law $\mathbb{P}^{x}$ of $\left(X_{t}^{x}\right)_{t \geq 0}$ started at $x$. The infinitesimal generator of $\mathcal{P}^{0}$ is a second-order differential operator of the form

$$
\mathcal{A}^{0} f(x)=\frac{1}{2} \sigma^{2}(x) f^{\prime \prime}(x)+b(x) f^{\prime}(x)
$$

with the domain $D\left(\mathcal{A}^{0}\right)=\left\{f \in C([0, \infty]) \cap C^{2}((0, \infty)): \mathcal{A}^{0} f \in C([0, \infty])\right\}$ if zero is an unattainable boundary. If zero is an instantaneously reflecting boundary, the Neumann-type boundary condition is additionally imposed at zero [Ethier and Kurtz (1986), page 367, equation (1.11) with $q_{0}=0$ ]. We also note that when zero and infinity are both natural boundaries, the semigroup leaves the space $C_{0}((0, \infty)) \subset C([0, \infty])$ of functions continuous on $(0, \infty)$ and having zero limits $\lim _{x \rightarrow 0} f(x)=0$ and $\lim _{x \rightarrow 0} f(x)=0$ invariant and is a Feller semigroup on it. If zero is not a natural boundary, while infinity is, then the semigroup is Feller on $C_{0}([0, \infty))$.

We next assume that our probability space supports a unit-mean exponential random variable $\mathcal{E} \sim \operatorname{Exp}(1)$ independent of the Brownian motion $B$ (and hence, of $X$ ). Define a random time $\zeta$

$$
\zeta:=\inf \left\{t \geq 0: \int_{0}^{t} k\left(X_{u}\right) d u \geq \mathcal{E}\right\}
$$

where $k(x) \geq 0$ is a given function assumed continuous on $(0, \infty)$. If zero is an instantaneously reflecting boundary, we assume that there is a finite limit $\lim _{x \rightarrow 0} k(x)<\infty$. If zero is unattainable, we do not make any assumptions about the behavior of $k(x)$ as $x \rightarrow 0$. Under these assumptions, $\int_{0}^{t} k\left(X_{u}\right) d u<$ $\infty$ a.s. for any initial condition $X_{0}=x>0$ [from our assumptions that $X$ does not explode to infinity, and that $k(x)$ is continuous on $(0, \infty)$ and has a finite limit at zero if zero is an attainable boundary for $X$ ]. Key examples of functions $k(x)$ of interest to us for credit risk applications are given in Examples 2.2-2.6 at the end of this section.

We denote by $\left(\mathcal{P}_{t}^{\beta}\right)_{t \geq 0}$ the Feynman-Kac semigroup associated with the positive continuous additive functional $\int_{0}^{t} \beta k\left(X_{u}\right) d u$ with $\beta>0$,

$$
\mathcal{P}_{t}^{\beta} f(x)=\mathbb{E}^{x}\left[e^{-\beta \int_{0}^{t} k\left(X_{u}\right) d u} f\left(X_{t}\right)\right] .
$$

Under our assumptions, it is a sub-Markovian-Feller semigroup on $C([0, \infty])$ with the generator

$$
\mathcal{A}^{\beta} f(x)=\mathcal{A}^{0} f(x)-\beta k(x) f(x)
$$

with the domain $D\left(\mathcal{A}^{\beta}\right) \subseteq D\left(\mathcal{A}^{0}\right)$. More precisely, $D\left(\mathcal{A}^{\beta}\right)=\{f \in C([0, \infty]) \cap$ $\left.C^{2}((0, \infty)): \mathcal{A}^{\beta} f \in C([0, \infty])\right\}$ if zero is an unattainable boundary for a diffu- 
sion with killing at the rate $\beta k(x)$; see Borodin and Salminen (2002), pages 16-17, for Feller's boundary classification of one-dimensional diffusions with killing. If zero is instantaneously reflecting for the diffusion with killing at the rate $\beta k(x)$, the Neumann-type boundary condition is imposed at zero [Ethier and Kurtz (1986), page 367, equation (1.11) with $q_{0}=0$ ].

We next associate to the random time $\zeta$ the event indicator process (onepoint process) $\left(D_{t}\right)_{t \geq 0}$ defined by

$$
D_{t}:=\mathbf{1}_{\{\zeta \leq t\}}, \quad t \geq 0,
$$

denote by $\mathbb{D}=\left(\mathcal{D}_{t}\right)_{t \geq 0}$ its (completed) natural filtration and define an enlarged filtration $\mathbb{G}=\left(\mathcal{G}_{t}\right)_{t \geq 0}$ with $\mathcal{G}_{t}=\mathcal{F}_{t}^{B} \vee \mathcal{D}_{t}$. This filtration is the smallest one which contains $\mathbb{F}^{B}$ and such that the random time $\zeta$ is a stopping time; cf. Jeanblanc, Yor and Chesney (2009), Section 7.3.3. From Jeanblanc, Yor and Chesney (2009), Proposition 5.9.1.1 and Remark 7.5.1.2, we observe that the filtrations $\mathbb{F}^{B}$ and $\mathbb{G}, \mathbb{F}^{B} \subset \mathbb{G}$, satisfy the $\mathbf{H}$-Hypothesis. As a result, any $\mathbb{F}^{B}$-local martingale is also a $\mathbb{G}$-local martingale.

We will now study the bi-variate process $\left(X_{t}, D_{t}\right)_{t \geq 0}$ of the state variable $X$ and the event indicator $D$. Given our assumptions, for any initial conditions $X_{0}=x>0$ and $D_{0}=d \in\{0,1\},(X, D)$ is a Markovian semimartingale taking values in $\mathbb{R}_{+} \times\{0,1\} \subset \mathbb{R}^{2}\left(D_{0}=1\right.$ corresponds to $\zeta=0$, and hence $D_{t}=1$ for all $t>0$ when $\left.D_{0}=1\right)$. We first characterize its Markovian nature. To this end, observe that any function $f(x, d) \in C([0, \infty] \times\{0,1\})$ can be written in the form

$$
f(x, d)=f_{1}(x)+(1-d)\left(f_{0}(x)-f_{1}(x)\right),
$$

where $f_{0}(x):=f(x, 0) \in C([0, \infty])$ and $f_{1}(x):=f(x, 1) \in C([0, \infty])$.

TheOREM 2.1 [Markovian characterization of $(X, D)$ ]. (i) The bi-variate process $(X, D)$ is a Feller process whose Feller semigroup $\left(\mathcal{P}_{t}\right)_{t \geq 0}$ acts on $f \in C([0, \infty] \times\{0,1\})$ according to

$$
\mathcal{P}_{t} f(x, d)=\mathcal{P}_{t}^{0} f_{1}(x)+(1-d) \mathcal{P}_{t}^{1}\left(f_{0}-f_{1}\right)(x),
$$

where $f_{0}(x)=f(x, 0) \in C([0, \infty]), f_{1}(x)=f(x, 1) \in C([0, \infty]),\left(\mathcal{P}_{t}^{0}\right)_{t>0}$ is the transition semigroup (2.2) of $X$ on $C([0, \infty])$ and $\left(\mathcal{P}_{t}^{1}\right)_{t>0}$ is the FeynmanKac semigroup (2.3) on $C([0, \infty])$.

(ii) The infinitesimal generator of the Feller semigroup $\left(\mathcal{P}_{t}\right)_{t \geq 0}$ is given by

$$
\begin{aligned}
\mathcal{A} f(x, d) & =\mathcal{A}^{0} f_{1}(x)+(1-d) \mathcal{A}^{1}\left(f_{0}-f_{1}\right)(x) \\
& =\mathcal{A}^{0} f(x, d)+(1-d) k(x)(f(x, 1)-f(x, 0)),
\end{aligned}
$$

where $\mathcal{A}^{0}$ and $\mathcal{A}^{1}$ are the generators of $\left(\mathcal{P}_{t}^{0}\right)_{t \geq 0}$ and $\left(\mathcal{P}_{t}^{1}\right)_{t \geq 0}$, respectively. 
(iii) If $f(x, d) \in D(\mathcal{A})$ [i.e., $f$ is of the form (2.4) with $f_{0}, f_{1} \in D\left(\mathcal{A}^{1}\right)$ ] and $(X, D)$ starts from $X_{0}=x>0$ and $D_{0}=d \in\{0,1\}$, then the process

$$
M_{t}^{f}:=f\left(X_{t}, D_{t}\right)-f(x, d)-\int_{0}^{t} \mathcal{A} f\left(X_{s}, D_{s}\right) d s
$$

is a $\mathbb{G}$-martingale.

Proof. (i) For all $0 \leq s<t$, we have

$$
\begin{aligned}
\mathbb{E}\left[f\left(X_{t}, D_{t}\right) \mid \mathcal{G}_{s}\right] & =\mathbb{E}\left[\left(1-D_{t}\right)\left(f_{0}-f_{1}\right)\left(X_{t}\right) \mid \mathcal{G}_{s}\right]+\mathbb{E}\left[f_{1}\left(X_{t}\right) \mid \mathcal{G}_{s}\right] \\
& =\left(1-D_{s}\right) \mathbb{E}\left[e^{-\int_{s}^{t} k\left(X_{u}\right) d u}\left(f_{0}-f_{1}\right)\left(X_{t}\right) \mid \mathcal{F}_{s}^{B}\right]+\mathbb{E}\left[f_{1}\left(X_{t}\right) \mid \mathcal{F}_{s}^{B}\right] \\
& =\left(1-D_{s}\right) \mathcal{P}_{t-s}^{1}\left(f_{0}-f_{1}\right)\left(X_{s}\right)+\mathcal{P}_{t-s}^{0} f_{1}\left(X_{s}\right) \\
& =\mathcal{P}_{t-s} f\left(X_{s}, D_{s}\right) .
\end{aligned}
$$

The first equality follows from the representation (2.4), the second equality is a standard result in intensity modeling in credit risk [e.g., Jeanblanc, Yor and Chesney (2009), Corollary 7.3.4.2, or Bielecki and Rutkowski (2004), Corollary 5.1.1], the third equality follows from the Markov property and time homogeneity of $X$. Since the operators $\left(\mathcal{P}_{t}^{0}\right)_{t \geq 0}$ and $\left(\mathcal{P}_{t}^{1}\right)_{t \geq 0}$ form Feller semigroups on $C([0, \infty])$, it is then immediate that the operators $\left(\mathcal{P}_{t}\right)_{t \geq 0}$ form a Feller semigroup on $C([0, \infty] \times\{0,1\})$. Thus, the bi-variate process $(X, D)$ is a Feller process whose semigroup action on $C([0, \infty] \times\{0,1\})$ is given by equation (2.5). (ii) The expression for the generator $\mathcal{A}$ follows from equation (2.5), given $\mathcal{A}^{0}$ and $\mathcal{A}^{1}$ are the generators of $\mathcal{P}^{0}$ and $\mathcal{P}^{1}$. Part (iii) follows from Ethier and Kurtz (1986), Proposition 1.7, page 162.

Since $X$ is a continuous semimartingale, and $D$ is a one-point point process, the bi-variate process $(X, D)$ is a special semimartingale. We can write Itô formula for functions of time and the bi-variate process in the useful form that separates the process $f\left(s, X_{s}, D_{s}\right)$ into a predictable finite variation process, a continuous local martingale that is the stochastic integral with respect to Brownian motion, and a discontinuous martingale that is the integral with respect to the compensated one-point process.

TheOREM 2.2 [Itô formula for $(X, D)]$. (i) The one-point point process $D$ has the following Doob-Meyer decomposition:

$$
D_{t}=A_{t}+M_{t}, \quad A_{t}=\int_{0}^{t}\left(1-D_{s}\right) k\left(X_{s}\right) d s, \quad M_{t}=D_{t}-A_{t},
$$

where $A$ is the predictable $\mathbb{G}$-compensator of $D$ [so that $\lambda_{t}^{\mathbb{G}}:=\left(1-D_{t}\right) k\left(X_{t}\right)$ is its $\mathbb{G}$-intensity] and $M$ is a $\mathbb{G}$-martingale. 
(ii) Suppose the semimartingale $(X, D)$ starts from $X_{0}=x>0$ and $D_{0}=$ $d \in\{0,1\}$. For any function $f(t, x, d)=f_{1}(t, x)+(1-d)\left(f_{0}(t, x)-f_{1}(t, x)\right)$ with $f_{i}(t, x) \in C^{1,2}\left(\mathbb{R}_{+} \times(0, \infty)\right)$ if zero is an unattainable boundary for the process $X$ or $f_{i}(t, x) \in C^{1,2}\left(\mathbb{R}_{+} \times[0, \infty)\right)$ if zero is attainable for $X$, the process $f\left(t, X_{t}, D_{t}\right)$ is a special $\mathbb{G}$-semimartingale with the following canonical decomposition into a predictable finite variation process, a continuous local martingale, and a purely discontinuous martingale,

$$
\begin{aligned}
f\left(t, X_{t}, D_{t}\right)= & f(0, x, d)+\int_{0}^{t}\left(\partial_{s}+\mathcal{A}\right) f\left(s, X_{s}, D_{s}\right) d s \\
& +\int_{0}^{t} \sigma\left(X_{s}\right) \partial_{x} f\left(s, X_{s}, D_{s}\right) d B_{s} \\
& +\int_{0}^{t}\left(1-D_{s-}\right)\left(f\left(s, X_{s}, 1\right)-f\left(s, X_{s}, 0\right)\right) d M_{s} .
\end{aligned}
$$

Proof. (i) This is a standard result; cf. Lemma 7.3.4.3(ii) in Jeanblanc, Yor and Chesney (2009), page 421.

(ii) Since $X$ is a nonnegative semimartingale, the functions $f_{i}(t, x)$ only need to be defined for $x \geq 0$. In order for all the terms in Itô's formula to be well defined, when $X$ is strictly positive, the functions $f_{i}$ need only be $C^{1,2}\left(\mathbb{R}_{+} \times(0, \infty)\right)$, while in the case when $X$ can hit zero $f_{i}$ and their first and second derivatives in $x$ and first derivatives in $t$ need to have finite limits as $x \rightarrow 0$, so that $f_{i} \in C^{1,2}\left(\mathbb{R}_{+} \times[0, \infty)\right)$. With these observations, this form of Itô's formula immediately follows from the form of Itô's formula for special semimartingales in Jacod (1979), Theorem 3.89, page 109.

EXAmple 2.2 (CIR intensity model, Example 2.1 continued). Assuming that $\kappa, \theta, \sigma>0$, the CIR diffusion has the gamma stationary density

$$
\pi(x)=\frac{a^{b} x^{b-1}}{\Gamma(b)} e^{-a x}, \quad b:=\frac{2 \kappa \theta}{\sigma^{2}}, \quad a:=\frac{2 \kappa}{\sigma^{2}} .
$$

That is, for all $x \in I$ and $a, b>0, \lim _{t \rightarrow \infty} P_{t}^{0}(x, d y)=\pi(y) d y$. With this choice of parameters, $\lim _{t \rightarrow \infty} \mathbb{E}^{x}\left[X_{t}\right]=\int_{I} y \pi(y) d y=\theta$, and $\theta$ is referred to as the long-run mean and $\kappa$ as the rate of mean reversion of the CIR state variable.

Let $k(x)=x$ in the CIR intensity model. Then, the $\mathbb{G}$-intensity of the stopping time $\zeta$ is $\lambda_{t}^{\mathbb{G}}=\left(1-D_{t}\right) X_{t}$, and the indicator process $D_{t}$ has a $\mathbb{G}$-compensator $A_{t}=\int_{0}^{t}\left(1-D_{s}\right) X_{s} d s$. If $D$ is interpreted as the default indicator, then (under the assumption of zero recovery) the instantaneous credit spread is equal to the $\mathbb{G}$-intensity $\lambda_{t}^{\mathbb{G}}$. The corresponding default intensity model goes back to Duffie and Singleton (1999). Since zero is either an entrance or an instantaneously reflecting boundary and infinity is 
a natural boundary, the CIR Feynman-Kac semigroup $\left(\mathcal{P}_{t}^{1}\right)_{t \geq 0}$ is a subMarkovian-Feller semigroup on $C_{0}([0, \infty))$ [and also on $C_{0}((0, \infty))$, when the Feller condition is satisfied and zero is a natural boundary]. It coincides with the pricing semigroup in the CIR interest rate model. Explicit expressions for the densities of the CIR transition semigroup $\left(\mathcal{P}_{t}^{0}\right)_{t \geq 0}$ and the CIR Feynman-Kac semigroup $\left(\mathcal{P}_{t}^{\beta}\right)_{t \geq 0}$ with $\beta>0$ are given in Section 6 .

EXAMPLE 2.3 (Reciprocal CIR intensity model). Let $X$ follow the CIR process as in Example 2.1 and assume that the Feller condition is satisfied, but take $k(x)=1 / x$ instead of $k(x)=x$. This choice leads to the reciprocal CIR intensity model. It was applied in credit modeling by Andreasen (2001). Applying Itô's formula to the process $Y_{t}=1 / X_{t}$ (justified when the Feller condition is satisfied, since in that case the process stays strictly positive), we obtain the SDE for $Y$,

$$
Y_{t}=y+\int_{0}^{t} \tilde{\kappa}\left(\tilde{\theta}-Y_{s}\right) Y_{s} d s-\int_{0}^{t} \sigma Y_{s}^{3 / 2} d B_{s}
$$

where $Y_{0}=y=1 / x$ and $\tilde{\kappa}=\kappa /\left(\kappa \theta-\sigma^{2}\right)$ and $\tilde{\theta}=\kappa \theta-\sigma^{2}$. This SDE has quadratic drift and the so-called $3 / 2$ volatility. When $\tilde{\theta}>0$ and $\tilde{\kappa}>0$, which requires $\kappa \theta>\sigma^{2}$ for the CIR process $X$, this SDE also appeared as the model for the instantaneous inflation rate in Cox, Ingersoll and Ross (1985) and as the model for the instantaneous nominal interest rate in Lewis (1994) and Ahn and Gao (1999) (the so-called 3/2 model). In this case the process has a stationary density

$$
\pi(y)=\frac{\alpha^{\beta}}{\Gamma(\beta)} y^{-\beta-1} e^{-\alpha / y} \quad \text { where } \alpha:=\frac{2 \tilde{\kappa} \tilde{\theta}}{\sigma^{2}}, \quad \beta:=\frac{2\left(\sigma^{2}+\tilde{\kappa}\right)}{\sigma^{2}} .
$$

The $\mathbb{G}$-intensity in this model is $\lambda_{t}^{\mathbb{G}}=\left(1-D_{t}\right) Y_{t}=\left(1-D_{t}\right) / X_{t}$, where $Y$ is the $3 / 2$-diffusion, or equivalently $X$ is the CIR diffusion. The semigroup $\left(\mathcal{P}^{1}\right)_{t \geq 0}$ can be obtained explicitly in this case and coincides with the pricing semigroup in the $3 / 2$ interest rate model.

EXAmPle 2.4 [Quadratic Ornstein-Uhlenbeck (OU) model]. Consider an $\operatorname{SDE}(2.1)$ with

$$
\sigma(x)=2 \sigma \sqrt{x}, \quad b(x)=2 \kappa(a+\theta \sqrt{x}-x)
$$

with $\sigma>0, \kappa>0, \theta \geq 0$, and $a=\sigma^{2} /(2 \kappa)$. This SDE is similar to the CIR $\mathrm{SDE}$, but has an extra term with $\sqrt{x}$ in the drift. Let $Y_{t}$ be the OU process solving the SDE $Y_{t}=y+\int_{0}^{t} \kappa\left(\theta-Y_{u}\right) d u+\sigma B_{t}$. Applying Itô's formula to the square of the OU process, $X_{t}=Y_{t}^{2}$, we verify that $X$ satisfies the SDE with the coefficients (2.7). The Feynman-Kac semigroup $\left(\mathcal{P}_{t}^{1}\right)_{t \geq 0}$ of the quadratic OU model coincides with the pricing semigroup in the quadratic OU interest rate model studied in Beaglehole and Tenney (1992) and Jamshidian (1996). 
EXAMPLE 2.5 [Carr and Linetsky (2006) JDCEV credit-equity model]. A jump-to-default extended constant elasticity of variance (JDCEV) diffusion of Carr and Linetsky (2006) models the pre-default stock price of a firm as the diffusion with

$$
\begin{aligned}
& \sigma(x)=a x^{\beta+1}, \quad b(x)=(r-q+k(x)) x, \\
& k(x)=b+c \sigma^{2}(x)=b+c a^{2} x^{2 \beta},
\end{aligned}
$$

where $a>0$ fixes the volatility scale, the constant elasticity of variance $\beta<0$ is assumed negative to capture the leverage effect (volatility of the stock price increases as the stock price falls), $r \geq 0$ is the risk-free rate, $q \geq 0$ is the dividend yield, and $k(x)$ is the function defining the default intensity in the JDCEV model, where $b \geq 0$ is the constant part and $c \geq 0$ is the sensitivity of the default intensity to the instantaneous variance of the stock price. $k(x) x$ is added to the drift to compensate for a jump to default to ensure that, under the risk-neutral measure, the discounted stock price with dividends reinvested and subject to default is a martingale. Thus, in the JDCEV model the stock price of a firm subject to default risk is $S_{t}=\left(1-D_{t}\right) X_{t}$, where $D_{t}$ is the default indicator (stock price drops to zero when the firm defaults on its debt). The $\mathbb{G}$-intensity is $\lambda_{t}^{\mathbb{G}}=\left(1-D_{t}\right)\left(b+c a^{2} X_{t}^{2 \beta}\right)$.

For any $a>0, \mu:=r-q+b \in \mathbb{R}, \beta<0$ and $c \in\left[(1 / 2+\beta)^{+}, \infty\right)$, the JDCEV SDE can be reduced to the CIR SDE as follows. Let $\left(Y_{t}\right)_{t \geq 0}$ be the unique strong solution of the CIR SDE with $Y_{0}=y>0$ and parameters satisfying $\kappa \theta>0$ and $\sigma>0$. For all $t \geq 0$ define a new process $X_{t}=\left(Y_{t}\right)^{1 /(2|\beta|)}$ with the initial condition $X_{0}=x=y^{1 /(2|\beta|)}>0$. Then by Itô's formula (the application is justified since $|\beta|>0$ ), the process $X_{t}$ solves the JDCEV SDE with $a=\frac{\sigma}{2|\beta|}, \mu=-\frac{\kappa}{2|\beta|}$, and $c=1 / 2+|\beta|\left(\frac{2 \kappa \theta}{\sigma^{2}}-1\right)$. Since we are only interested in nonnegative default intensities, we impose the condition $1 / 2+|\beta|\left(\frac{2 \kappa \theta}{\sigma^{2}}-1\right) \geq 0$. When the CIR process $Y$ satisfies Feller's condition, $2 \kappa \theta \geq \sigma^{2}$, the JDCEV parameter satisfies $c \in[1 / 2, \infty)$. In this case, the boundary at zero is entrance for both the CIR and JDCEV diffusions. When $2 \kappa \theta \in\left(0, \sigma^{2}\right)$, the resulting JDCEV parameter satisfies $c \in\left((1 / 2+\beta)^{+}, 1 / 2\right)$. In this case, the boundary at zero is instantaneously reflecting for both CIR and JDCEV. In both cases, the killing rate $k$ reduces to $k(x)=b+c a^{2} / y$ in terms of the CIR variable $y$, and hence the JDCEV FK semigroup $\left(\mathcal{P}_{t}^{1}\right)_{t \geq 0}$ reduces to the Feynman-Kac semigroup in the reciprocal CIR model of Example 2.3 (with the constant $b$ added). Finally, we remark that while the JDCEV diffusion can also be defined when $-1 / 2<\beta<0$ and $c \in\left[0,(1 / 2+\beta)^{+}\right)$ (in this case zero is an exit boundary), it cannot be reduced to the CIR diffusion for this set of parameters. Since in this paper we do not consider exit boundaries, we are not concerned with this case in the present paper.

EXAMPLE 2.6 [Linetsky (2006) credit-equity model]. Also in the context of credit-equity models, Linetsky (2006) studies an extension of the Black- 
Scholes-Merton (BSM) model with bankruptcy where the killing rate is a negative power of the state variable. The pre-default dynamics of the stock price are determined by

$$
\sigma(x)=\sigma x, \quad b(x)=(r-q+k(x)) x, \quad k(x)=\alpha x^{-p},
$$

where $\sigma>$ is the constant volatility, $r \geq 0$ is the risk-free rate, $q \geq 0$ is the dividend yield and $k(x)$ is the killing rate specified to be a negative power of the stock price with $\alpha>0$ and $p>0$. As in Example 2.5, the killing rate $k(x)$ is added into the drift to compensate for the jump to default that makes the stock price worthless in default. By specifying $k(x)$ to be the negative power of the stock price, this model is able to exhibit implied volatility skews in stock option prices, with the parameters $\alpha$ and $p$ of the killing rate specification controlling the slope of the skew, thus establishing a link between implied volatility skews and credit spreads (as the stock price drops, the implied volatility and the probability of default increase). In this case, the stock price is $S_{t}=\left(1-D_{t}\right) X_{t}$ and the $\mathbb{G}$-intensity of default in this model is $\lambda_{t}^{\mathbb{G}}=\left(1-D_{t}\right) \alpha X_{t}^{p}$.

3. The subordinated diffusion default intensity model. We next assume that our probability space $(\Omega, \mathcal{F}, \mathbb{P})$ also supports a Lévy subordinator $\left(\mathcal{T}_{t}\right)_{t \geq 0}$ independent of both the Brownian motion $B$ and the exponential random variable $\mathcal{E}$ and thus is independent of the bi-variate process $(X, D)$. Recall that a Lévy subordinator is a nondecreasing Lévy process, that is, a Lévy process with one-sided positive jumps and nonnegative drift and no diffusion component. The Laplace transform of a Lévy subordinator $\left(\mathcal{T}_{t}\right)_{t \geq 0}$ is given by the Lévy-Khintchine formula

$$
\begin{aligned}
\mathbb{E}\left[e^{-\lambda \mathcal{T}_{t}}\right]=\int_{[0, \infty)} e^{-\lambda s} \pi_{t}(d s) & =e^{-t \phi(\lambda)} \\
\text { with } \phi(\lambda) & =\gamma \lambda+\int_{(0, \infty)}\left(1-e^{-\lambda s}\right) \nu(d s) .
\end{aligned}
$$

Here $\pi_{t}(d s)$ is the transition kernel, $\phi(\lambda)$ is a Lévy exponent, $\gamma \geq 0$ is the nonnegative drift and $\nu(d s)$ is a Lévy measure of the subordinator that satisfies the integrability condition $\int_{(0, \infty)}(s \wedge 1) \nu(d s)<\infty$ [standard references on subordinators are Bertoin (1996, 1999), Sato (1999) and Schilling, Song and Vondraček (2010)].

ExAmple 3.1 (Tempered stable and related subordinators). A family of subordinators important in financial applications is defined by the following three-parameter family of Lévy measures:

$$
\nu(d s)=C s^{-\alpha-1} e^{-\eta s} d s
$$

with $C>0, \eta>0$, and $\alpha<1$. For $\alpha \in(0,1)$ these are the so-called tempered stable subordinators [exponentially dampened counterparts of the $\alpha$-stable 
subordinators with $\left.\nu(d s)=C s^{-\alpha-1} d s\right]$. The special case $\alpha=1 / 2$ is the $i n$ verse Gaussian process [Barndorff-Nielsen (1998)]. The limiting case $\alpha=0$ is the gamma process [Madan, Carr and Chang (1998)]. Subordinators with $\alpha \in[0,1)$ are infinite activity processes. Subordinators with $\alpha<0$ are compound Poisson processes with gamma distributed jump sizes. The compound Poisson process with the Lévy measure $\nu(d s)=\omega \eta e^{-\eta s} d s$ with exponential jumps is a special case with $\alpha=-1$ and $C=\omega \eta$, where $\omega$ is the jump arrival rate, and $1 / \eta$ is the mean of the exponential jump size distribution. The Laplace exponent is given by

$$
\phi(\lambda)= \begin{cases}\gamma \lambda-C \Gamma(-\alpha)\left[(\lambda+\eta)^{\alpha}-\eta^{\alpha}\right], & \alpha \neq 0, \\ \gamma \lambda+C \ln (1+\lambda / \eta), & \alpha=0,\end{cases}
$$

where $\Gamma(x)$ is the gamma function.

We now time change the bi-variate process $(X, D)$ of the previous section with a subordinator $\mathcal{T}$. That is, we define a new bi-variate process $\left(X_{t}^{\phi}, D_{t}^{\phi}\right)_{t \geq 0}$ by

$$
X_{t}^{\phi}:=X(\mathcal{T}(t)), \quad D_{t}^{\phi}:=D(\mathcal{T}(t))
$$

and assume that $\left(D_{t}^{\phi}\right)_{t \geq 0}$ is the default indicator process (i.e., the default time is the first time $D^{\bar{\phi}}$ is equal to one), and $X^{\phi}$ is the state variable that models the credit health of the obligor. We also define the time changed filtration as follows. Define an inverse subordinator process as the right inverse $\left(L_{t}:=\inf \left\{s \geq 0: \mathcal{T}_{s}>t\right\}\right)_{t \geq 0}$. Since $\mathcal{T}$ is cádlág, so is $L$. Let $\mathbb{L}=\left(\mathcal{L}_{t}\right)_{t \geq 0}$ be its completed natural filtration. Let $\mathbb{H}=\left(\mathcal{H}_{t}\right)_{t \geq 0}$ denote the enlarged filtration with $\mathcal{H}_{t}=\mathcal{G}_{t} \vee \mathcal{L}_{t}$, where $\mathcal{G}_{t}$ refers to the filtration $\mathbb{G}=\left(\mathcal{G}_{t}\right)_{t \geq 0}$ of Section 2 . Then $\left(\mathcal{T}_{t}\right)_{t>0}$ is an increasing family of $\mathbb{H}$-stopping times, and we can define the time changed filtration $\mathbb{H}^{\phi}=\left(\mathcal{H}_{t}^{\phi}\right)_{t \geq 0}$ by $\mathcal{H}_{t}^{\phi}=\mathcal{H}_{\mathcal{T}_{t}}$ for all $t \geq 0$. The time changed bi-variate process $\left(X_{t}^{\phi}, D_{t}^{\bar{\phi}}\right)_{t \geq 0}$ is obviously $\mathbb{H}^{\phi}$-adapted and cádlág.

Proposition 3.1. The process $\left(X_{t}^{\phi}, D_{t}^{\phi}\right)_{t>0}$ is an $\mathbb{H}^{\phi}$-semimartingale.

Proof. Since $\left(X_{t}^{\phi}, D_{t}^{\phi}\right)_{t>0}$ is a time change of a semimartingale $(X, D)$, it is a semimartingale by Corollary 10.12 in Jacod (1979), page 315.

We will now investigate its properties. In particular, we show that $\left(X_{t}^{\phi}\right.$, $\left.D_{t}^{\phi}\right)_{t>0}$ is a Feller process with the Feller semigroup on $C([0, \infty] \times\{0,1\})$, explicitly compute its infinitesimal generator, obtain its predictable semimartingale characteristics and give Itô's formula.

We first recall some key results about the subordination in the sense of Bochner of semigroups of operators in Banach spaces. The procedure of 
subordination goes back to Bochner (1949). The expression for the generator constitutes the Phillips theorem [Phillips (1952)]. The formulation below is reproduced from Sato (1999), Theorem 32.1.

Theorem 3.1 (Subordination in the sense of Bochner; Phillips theorem). Let $\left(\mathcal{T}_{t}\right)_{t \geq 0}$ be a subordinator with Lévy measure $\nu$, drift $\gamma$, Laplace exponent $\phi(\lambda)$ and transition function $\pi_{t}(d s)$. Let $\left(\mathcal{P}_{t}\right)_{t \geq 0}$ be a strongly continuous contraction semigroup of linear operators on a Banach space $\mathfrak{B}$ with infinitesimal generator $\mathcal{A}$.

(i) Define

$$
\mathcal{P}_{t}^{\phi} f(x)=\int_{[0, \infty)} \mathcal{P}_{s} f(x) \pi_{t}(d s), \quad t \geq 0, f \in \mathfrak{B} .
$$

Then $\left(\mathcal{P}_{t}^{\phi}\right)_{t \geq 0}$ is a strongly continuous contraction semigroup of linear operators on $\mathfrak{B}$ called subordinate semigroup of $\left(\mathcal{P}_{t}\right)_{t \geq 0}$ with respect to the subordinator $\left(\mathcal{T}_{t}\right)_{t \geq 0}$.

(ii) Denote the infinitesimal generator of $\left(\mathcal{P}_{t}^{\phi}\right)_{t \geq 0}$ by $\mathcal{A}^{\phi}$. Then the domain of $\mathcal{A}$ is a core of $\mathcal{A}^{\phi}$ and

$$
\mathcal{A}^{\phi} f=\gamma \mathcal{A} f+\int_{(0, \infty)}\left(\mathcal{P}_{s} f-f\right) \nu(d s), \quad f \in \operatorname{Dom}(\mathcal{A}) .
$$

We will need the following corollary.

Corollary 3.1. If $\left(\mathcal{P}_{t}\right)_{t \geq 0}$ is a Feller semigroup on $C([0, \infty])$, then the subordinate semigroup $\left(\mathcal{P}_{t}^{\phi}\right)_{t \geq 0}$ is also a Feller semigroup on $C([0, \infty])$.

Proof. The space $C([0, \infty])$ consists of continuous functions on $[0, \infty]$ or, equivalently, continuous functions on $(0, \infty)$ with finite limits at 0 and $\infty$. A strongly continuous contraction semigroup on $C([0, \infty])$ is Feller if it is positivity preserving. Suppose $\left(\mathcal{P}_{t}\right)_{t \geq 0}$ is Feller on $C([0, \infty])$. Then $\left(\mathcal{P}_{t}^{\phi}\right)_{t \geq 0}$ is a strongly continuous contraction semigroup on $C([0, \infty])$ by Theorem 3.1 (i) with $\mathfrak{B}=C([0, \infty])$. Since Bochner's integral in equation (3.3) is positivity preserving, for all $u \in C([0, \infty])$ such that $0 \leq u \leq 1$, we have $0 \leq \mathcal{P}_{t}^{\phi} u \leq 1$. Thus $\left(\mathcal{P}_{t}^{\phi}\right)_{t \geq 0}$ is positivity preserving and, hence, Feller on $C([0, \infty])$.

Recall that $I=(0, \infty)$ if 0 is unattainable and $I=[0, \infty)$ if 0 is reflecting. Under our assumptions, the transition kernels of the semigroups $\left(\mathcal{P}_{t}^{\beta}\right)_{t \geq 0}$ have densities with respect to the Lebesgue measure, $P_{t}^{\beta}(x, d y)=$ $p^{\beta}(t, x, y) d y$, where $p^{\beta}(t, x, y)$ are jointly continuous in $t, x, y$. This follows from the fact that any one-dimensional diffusion has a density with respect 
to the speed measure that is jointly continuous in $t, x, y$; cf. McKean (1956) or Borodin and Salminen (2002), page 13. Under our assumptions, the speed measure is absolutely continuous with respect to the Lebesgue measure [cf. Borodin and Salminen (2002), page 17], and hence the semigroups have densities with respect to the Lebesgue measure. For $\beta=0$ the density is the proper probability density on $I, P_{t}^{0}(x, I)=\int_{I} p^{1}(t, x, y) d y=1$ for each $x \in I$. For $\beta>0$, the density is generally defective, $P_{t}^{\beta}(x, I)=\int_{I} p^{\beta}(t, x, y) d y \leq 1$. For notational convenience we extend the densities from $I$ to $\mathbb{R}$ by setting $p^{\beta}(t, x, y) \equiv 0$ for $y<0$ for all $x \in I$ and $t>0$. We are now ready to give the Markovian characterization of the time-changed process $\left(X^{\phi}, D^{\phi}\right)$ defined by (3.2) based on Phillips Theorem 3.1 and Corollary 3.1.

TheOrem 3.2 [Markovian characterization of $\left(X^{\phi}, D^{\phi}\right)$ ]. (i) The bivariate process $\left(X^{\phi}, D^{\phi}\right)$ is a Feller process with the Feller semigroup $\left(\mathcal{P}_{t}^{\phi}\right)_{t \geq 0}$ acting on $f \in C([0, \infty] \times\{0,1\})$ by

$$
\mathcal{P}_{t}^{\phi} f(x, d)=\mathcal{P}_{t}^{0, \phi} f_{1}(x)+(1-d) \mathcal{P}_{t}^{1, \phi}\left(f_{0}-f_{1}\right)(x),
$$

where $f_{0}(x)=f(x, 0) \in C([0, \infty]), f_{1}(x)=f(x, 1) \in C([0, \infty])$ and $\left(\mathcal{P}_{t}^{0, \phi}\right)_{t \geq 0}$ and $\left(\mathcal{P}_{t}^{1, \phi}\right)_{t \geq 0}$ are Feller semigroups obtained by subordination in the sense of Bochner from Feller semigroups $\left(\mathcal{P}_{t}^{0}\right)_{t \geq 0}$ and $\left(\mathcal{P}_{t}^{1}\right)_{t \geq 0}$.

(ii) The infinitesimal generator $\mathcal{A}^{\phi}$ of the Feller semigroup $\left(\mathcal{P}_{t}^{\phi}\right)_{t \geq 0}$ has the following representation:

$$
\mathcal{A}^{\phi} f(x, d)=\mathcal{A}^{0, \phi} f_{1}(x)+(1-d) \mathcal{A}^{1, \phi}\left(f_{0}-f_{1}\right)(x),
$$

$$
f_{0}, f_{1} \in \operatorname{Dom}\left(\mathcal{A}^{1}\right)
$$

where $\mathcal{A}^{\beta, \phi}, \beta \in\{0,1\}$, are generators of $\left(\mathcal{P}_{t}^{\beta, \phi}\right)_{t \geq 0}$.

(iii) The generator $\mathcal{A}^{\beta, \phi}$ has the following Lévy-Khintchine-type representations with state-dependent coefficients

$$
\begin{aligned}
\mathcal{A}^{\beta, \phi} f(x)= & \frac{1}{2} \gamma \sigma^{2}(x) f^{\prime \prime}(x)+b^{\beta, \phi}(x) f^{\prime}(x)-k^{\phi}(x) f(x) \\
& +\int_{\mathbb{R}}\left(f(x+y)-f(x)-\mathbf{1}_{\{|y| \leq 1\}} y f^{\prime}(x)\right) \pi^{\beta, \phi}(x, y) d y
\end{aligned}
$$

for all $f \in D\left(\mathcal{A}^{\beta}\right)$, where the state-dependent Lévy density $\pi^{\beta, \phi}(x, y)$ is defined for all $y \neq 0$ by

$$
\pi^{\beta, \phi}(x, y)=\int_{(0, \infty)} p^{\beta}(s, x, x+y) \nu(d s),
$$

and satisfies the integrability condition $\int_{\mathbb{R}}\left(|y|^{2} \wedge 1\right) \pi^{\beta, \phi}(x, y) d y<\infty$ for each $x \in I$ [recall that we extended $p(t, x, y)$ to $\mathbb{R}$ by setting $p(t, x, y)=0$ for $y<0$ ], 
the drift with respect to the truncation function $x \mathbf{1}_{\{|x| \leq 1\}}$ is given by

$$
b^{\beta, \phi}(x)=\gamma b(x)+\int_{(0, \infty)}\left(\int_{\{|y| \leq 1\}} y p^{\beta}(s, x, x+y) d y\right) \nu(d s),
$$

and the killing rate is given by

$$
k^{\phi}(x)=\gamma \beta k(x)+\int_{(0, \infty)}\left(1-P_{s}^{\beta}(x, I)\right) \nu(d s),
$$

where $P_{s}^{\beta}(x, I)=\int_{I} p^{1}(s, x, y) d y$.

(iv) If $f(x, d) \in D(\mathcal{A})$ [i.e., $f$ is of the form (2.4) with $f_{0}, f_{1} \in D\left(\mathcal{A}^{1}\right)$ ] and $\left(X^{\phi}, D^{\phi}\right)$ starts with $X_{0}^{\phi}=x>0$ and $D_{0}^{\phi}=d \in\{0,1\}$, then the process

$$
M_{t}^{f}:=f\left(X_{t}^{\phi}, D_{t}^{\phi}\right)-f(x, d)-\int_{0}^{t} \mathcal{A}^{\phi} f\left(X_{s}^{\phi}, D_{s}^{\phi}\right) d s
$$

is an $\mathbb{H}^{\phi}$-martingale.

Proof. (i) The semigroup $\left(\mathcal{P}_{t}^{\phi}\right)_{t \geq 0}$ of $\left(X^{\phi}, D^{\phi}\right)$ is Feller by Corollary 3.1. The explicit representation (3.5) for the semigroup results from combining equation (2.5) with (3.3) of Theorem 3.1.

(ii) Representation (3.6) for the generator $\mathcal{A}^{\phi}$ in terms of generators of subordinate semigroups $\left(\mathcal{P}_{t}^{\beta, \phi}\right)_{t \geq 0}$ follows from (3.4).

(iii) Explicit representation (3.7) for the generator $\mathcal{A}^{\beta, \phi}$ is shown as follows. We start by observing that by Theorem 4.5 of McKean (1956) for each $x \in I$ the density $p^{\beta}(t, x, y)$ satisfies the following estimates:

$$
\begin{aligned}
\int_{\{|y-x|>1\}} p^{\beta}(t, x, y) d y & \leq C_{1} t, \\
\int_{\{|y-x| \leq 1\}}(y-x)^{2} p^{\beta}(t, x, y) d y & \leq C_{2} t, \\
\left|\int_{\{|y-x| \leq 1\}}(y-x) p^{\beta}(t, x, y) d y\right| & \leq C_{3} t, \\
1-\int_{I} p^{\beta}(t, x, y) d y & \leq C_{4} t .
\end{aligned}
$$

For each $x \in I$ write

$$
\begin{aligned}
\mathcal{P}_{s}^{\beta} f(x)-f(x)= & \int_{\mathbb{R}}\left(f(x+y)-f(x)-\mathbf{1}_{\{|y| \leq 1\}} y f^{\prime}(x)\right) p^{\beta}(s, x, x+y) d y \\
& +\left(\int_{\{|y| \leq 1\}} y p^{\beta}(s, x, x+y) d y\right) f^{\prime}(x)-\left(1-P_{s}^{\beta}(x, I)\right) f(x) .
\end{aligned}
$$


Substitute the result into the Phillips representation (3.4) of the generator of the subordinate semigroup, integrate term-by-term against the Lévy measure $\nu(d s)$ of the subordinator and interchange the integration in $y$ and in $s$ in the first of the three integrals. The result yields the representation (3.7)-(3.10). These operations are justified and the three resulting integrals are well defined due to estimates (3.12)-(3.15) and the integrability of the Lévy measure of the subordinator, $\int_{(0, \infty)}(1 \wedge s) \nu(d s)<\infty$. Specifically, estimates (3.12) and (3.13) ensure that the application of Fubini's theorem to interchange the integrations in $s$ and $y$ is justified, and the resulting integral in (3.7) is well defined for each $f \in D\left(\mathcal{A}^{\beta}\right)$, as they ensure that the measure $\pi^{\beta, \phi}(x, y) d y$ with the density (3.8) is a Lévy measure for each $x \in I$ [it is similar to Sato (1999), pages 200-201, proof that (30.8) is the Lévy measure of the subordinate Lévy process]. Estimate (3.14) ensures that the integral in (3.9) is well defined [it is similar to Sato (1999), proof that the drift (30.9) of the subordinate Lévy process is well defined]. Finally, estimate (3.15) ensures that the integral in (3.10) is well defined due to the integrand tending to zero at the rate $s$ as $s \rightarrow 0$.

Part (iv) follows from Ethier and Kurtz (1986), Proposition 1.7, page 162.

To obtain predictable characteristics of the semimartingale $\left(X^{\phi}, D^{\phi}\right)$ from the explicit form of the Feller generator $\mathcal{A}^{\phi}$, it is convenient to first re-write the generator in the following equivalent form.

COROLlary 3.2 (Alternative representation of the generator $\mathcal{A}^{\phi}$ ). The generator $\mathcal{A}^{\phi}$ admits the following alternative representation:

$\mathcal{A}^{\phi} f(x, d)$

$$
\begin{aligned}
& =\frac{1}{2} \gamma \sigma^{2}(x) \partial_{x}^{2} f(x, d)+b^{0, \phi}(x) \partial_{x} f(x, d)+(1-d) k^{\phi}(x) \partial_{d} f(x, d) \\
& +\int_{\mathbb{R}^{2}}\left(f(x+y, d+z)-f(x, d)-y \mathbf{1}_{\{|y| \leq 1\}} \partial_{x} f(x, d)-z \partial_{d} f(x, d)\right) \\
& \quad \times \Pi^{\phi}(x, d ; d y d z)
\end{aligned}
$$

where

$$
\begin{aligned}
\Pi^{\phi}(x, d & ; d y d z) \\
= & (1-d) \gamma k(x) \delta_{0}(d y) \delta_{1}(d z) \\
& +\left[\pi^{0, \phi}(x, y)-(1-d)\left(\pi^{0, \phi}(x, y)-\pi^{1, \phi}(x, y)\right)\right] d y \delta_{0}(d z) \\
& +(1-d)\left(\pi^{0, \phi}(x, y)-\pi^{1, \phi}(x, y)\right) d y \delta_{1}(d z),
\end{aligned}
$$

where $\pi^{\beta, \phi}(x, y)$ are the Lévy densities defined in equation (3.8) with $\beta=0,1$, and $\delta_{a}$ is the Dirac measure charging a. 
Proof. Denote the operator defined by equations (3.6)-(3.10) by $\hat{\mathcal{A}}^{\phi}$. We need to show that $\hat{\mathcal{A}}^{\phi} f(x, d)=\mathcal{A}^{\phi} f(x, d)$ for all $x \in I$ and $d \in\{0,1\}$, where $\mathcal{A}^{\phi}$ is the operator in equation (3.16). The case with $d=1$ is immediate, $\hat{\mathcal{A}}^{\phi} f(x, 1)=\mathcal{A}^{\phi} f(x, 1)=\mathcal{A}^{0, \phi} f(x, 1)$. Next consider the case $d=0$. From (3.6) we have

$$
\begin{aligned}
\hat{\mathcal{A}}^{\phi} f(x, 0)= & \frac{1}{2} \gamma \sigma^{2}(x) \partial_{x}^{2} f(x, 0) \\
& +b^{1, \phi}(x) \partial_{x} f(x, 0)+\left(b^{0, \phi}(x)-b^{1, \phi}(x)\right) \partial_{x} f(x, 1) \\
& +k^{\phi}(x)(f(x, 1)-f(x, 0)) \\
& +\int_{\mathbb{R}}\left(f(x+y, 0)-f(x, 0)-\mathbf{1}_{\{|y| \leq 1\}} y \partial_{x} f(x, 0)\right) \pi^{1, \phi}(x, y) d y \\
& +\int_{\mathbb{R}}\left(f(x+y, 1)-f(x, 1)-\mathbf{1}_{\{|y| \leq 1\}} y \partial_{x} f(x, 1)\right) \\
& \quad \times\left(\pi^{0, \phi}(x, y)-\pi^{1, \phi}(x, y)\right) d y .
\end{aligned}
$$

The last integral can be written as

$$
\begin{aligned}
\int_{\mathbb{R}}\left(f(x+y, 1)-f(x, 1)-\mathbf{1}_{\{|y| \leq 1\}} y \partial_{x} f(x, 1)\right)\left(\pi^{0, \phi}(x, y)-\pi^{1, \phi}(x, y)\right) d y \\
=\int_{\mathbb{R}}(f(x+y, 1)-f(x, 0)+f(x, 0)-f(x, 1) \\
\left.\quad-\mathbf{1}_{\{|y| \leq 1\}} y\left(\partial_{x} f(x, 1)-\partial_{x} f(x, 0)+\partial_{x} f(x, 0)\right)\right) \\
\quad \times\left(\pi^{0, \phi}(x, y)-\pi^{1, \phi}(x, y)\right) d y \\
=\int_{\mathbb{R}}\left(f(x+y, 1)-f(x, 0)-\mathbf{1}_{\{|y| \leq 1\}} y \partial_{x} f(x, 0)-\partial_{d} f(x, 0)\right) \\
\quad \times\left(\pi^{0, \phi}(x, y)-\pi^{1, \phi}(x, y)\right) d y \\
\quad-\left(\partial_{x} f(x, 1)-\partial_{x} f(x, 0)\right) \int_{\mathbb{R}} \mathbf{1}_{\{|y| \leq 1\}} y\left(\pi^{0, \phi}(x, y)-\pi^{1, \phi}(x, y)\right) d y .
\end{aligned}
$$

From equation (3.9) observe that

$$
\int_{\mathbb{R}} \mathbf{1}_{\{|y| \leq 1\}} y\left(\pi^{0, \phi}(x, y)-\pi^{1, \phi}(x, y)\right) d y=b^{0, \phi}(x)-b^{1, \phi}(x) .
$$

Substituting this result into (3.19) and substituting the result into (3.18) and comparing with (3.16)-(3.17), we establish that $\hat{\mathcal{A}}^{\phi} f(x, 0)=\mathcal{A}^{\phi} f(x, 0)$. 
Next we are ready to give semimartingale characterization of $\left(X^{\phi}, D^{\phi}\right)$. For the definition of predictable characteristics of a semimartingale, see Jacod and Shiryaev (2002), page 76 .

TheOREM 3.3 [Semimartingale characterization of $\left(X^{\phi}, D^{\phi}\right)$ ]. (i) The bi-variate $\mathbb{H}^{\phi}$-semimartingale $\left(X^{\phi}, D^{\phi}\right)$ has the following predictable characteristics. The predictable quadratic variation of the continuous local martingale component $X_{t}^{\phi, c}$ is

$$
C_{t}^{X^{\phi} X^{\phi}}=\int_{0}^{t} \gamma \sigma^{2}\left(X_{s}^{\phi}\right) d s
$$

$\left(C_{t}^{D^{\phi} D^{\phi}}=0\right.$ and $C_{t}^{X^{\phi} D^{\phi}}=0$ since $D^{\phi}$ is purely discontinuous). The predictable process of finite variation associated with the truncation function $\left(h^{X^{\phi}}(x, d)=x \mathbf{1}_{\{|x| \leq 1\}}, h^{D^{\phi}}(x, d)=d\right)$ is

$$
B_{t}^{X^{\phi}}=\int_{0}^{t} b^{0, \phi}\left(X_{s}^{\phi}\right) d s, \quad B_{t}^{D^{\phi}}=\int_{0}^{t}\left(1-D_{s}^{\phi}\right) k^{\phi}\left(X_{s}^{\phi}\right) d s,
$$

where the function $b^{0, \phi}(x)$ is defined in equation (3.9), and $k^{\phi}\left(X_{s}^{\phi}\right)$ is defined in equation (3.10). The compensator of the random measure $\mu(\omega ; d t, d y d z)$ associated to the jumps of $\left(X^{\phi}, D^{\phi}\right)$ is a predictable random measure on $\mathbb{R}_{+} \times\left(\mathbb{R}^{2} \backslash\{(0,0)\}\right)$,

$$
\nu(\omega ; d t, d y d z)=\Pi^{\phi}\left(X_{t-}^{\phi}, D_{t-}^{\phi} ; d y d z\right) d t
$$

with the measure $\Pi^{\phi}(x, d ; d y d z)$ given by equation (3.17).

(ii) The Lévy-Itô canonical representation of $X^{\phi}$ with respect to the truncation function $x \mathbf{1}_{\{|x| \leq 1\}}$ is

$$
\begin{aligned}
X_{t}^{\phi}= & +B_{t}^{X^{\phi}}+X_{t}^{\phi, c} \\
& +\int_{0}^{t} \int_{\mathbb{R}} y \mathbf{1}_{\{|y| \leq 1\}}\left(\mu^{X^{\phi}}(d s, d y)-\nu^{X^{\phi}}(d s, d y)\right) \\
& +\int_{0}^{t} \int_{\mathbb{R}} y \mathbf{1}_{\{|y|>1\}} \mu^{X^{\phi}}(d s, d y),
\end{aligned}
$$

where the compensator of the random measure $\mu^{X^{\phi}}(\omega ; d t, d y)$ associated to the jumps of $X^{\phi}$ is a predictable random measure on $\mathbb{R}_{+} \times(\mathbb{R} \backslash\{0\})$,

$$
\nu^{X^{\phi}}(\omega ; d t, d y)=\pi^{0, \phi}\left(X_{t-}^{\phi}, y\right) d y d t
$$

where $\pi^{0, \phi}(x, y)$ is defined in equation (3.8).

(iii) The Doob-Meyer decomposition of $D_{t}^{\phi}$ is

$$
D_{t}^{\phi}=A_{t}^{D^{\phi}}+M_{t}^{\phi}
$$


with the martingale $M_{t}^{\phi}=D_{t}^{\phi}-A_{t}^{D^{\phi}}$ and the predictable compensator $A_{t}^{D^{\phi}}=$ $B_{t}^{D^{\phi}}$ given in equation (3.20), so that the $\mathbb{H}^{\phi}$-intensity is $\lambda_{t}^{\mathbb{H}^{\phi}}=\left(1-D_{t}^{\phi}\right) k^{\phi}\left(X_{t}^{\phi}\right)$.

Proof. (i) By Theorem 2.42 of Jacod and Shiryaev (2002), page 86, the following two statements are equivalent: (i) the $n$-dimensional semimartingale $Z$ admits characteristics $(B, C, \nu)$ with respect to the truncation function $h$, and (ii) for each bounded function $f$ of class $C^{2}$ the process [using notation of equation (2.43) in Jacod and Shiryaev (2002), page 86]

$$
\begin{gathered}
f(Z)-f\left(Z_{0}\right)-\sum_{i \leq n} \partial_{i} f\left(Z_{-}\right) \bullet B^{i}-\frac{1}{2} \sum_{i, j \leq n} \partial_{i} \partial_{j} f\left(Z_{-}\right) \bullet C^{i j} \\
-\left(f\left(Z_{-}+z\right)-f\left(Z_{-}\right)-\sum_{i \leq n} h^{i}(z) \partial_{i} f\left(Z_{-}\right)\right) \star \nu
\end{gathered}
$$

is a local martingale. In our case $Z=\left(X^{\phi}, D^{\phi}\right)$ is a two-dimensional semimartingale such that for any $f \in D(\mathcal{A})$ the process (3.23) is a martingale. Substituting expression (3.16) for the generator into (3.11), we immediately identify the characteristics of $\left(X^{\phi}, D^{\phi}\right)$ since the characteristics are unique (up to a null set).

(ii) The result is shown by observing that $X^{\phi}$ is itself one-dimensional Markov with the generator $\mathcal{A}^{0, \phi}$ given by (3.7) with $\beta=0$, and identifying its predictable characteristics $\left(B^{X^{\phi}}, C^{X^{\phi} X^{\phi}}, \nu^{X^{\phi}}\right)$ with $\nu^{X^{\phi}}$ given by $(3.22)$ from the generator $\mathcal{A}^{0, \phi}$, as we did in (i) for the bi-variate process. The canonical representation of $X^{\phi}$ is then immediate by Theorem 2.34 of Jacod and Shiryaev (2002), page 84.

(iii) Immediate by Theorem 3.15 of Jacod and Shiryaev (2002) (the onepoint point process $D^{\phi}$ is a class $D$ submartingale) and the fact that $D^{\phi}=$ $B^{D^{\phi}}+M^{\phi}$ is the canonical decomposition of the special semimartingale $D^{\phi}$ [Proposition 2.29(a) of Jacod and Shiryaev (2002)].

From Theorem 3.3, we see that $\left(X^{\phi}, D^{\phi}\right)$ is a Markovian Itô semimartingale or Itô process in the terminology of Çinlar et al. (1980), page 165. In particular, when $\gamma>0, X^{\phi}$ is an Itô jump-diffusion with the continuous local martingale component with quadratic variation $\gamma \int_{0}^{t} \sigma^{2}\left(X_{s}\right) d s$ and with jumps with the predictable compensator (3.22). When $\gamma=0, X^{\phi}$ is a purejump process. Recall that every Itô semimartingale can be represented as a solution of a stochastic differential equation driven by a standard Brownian motion, Lebesgue measure and a Poisson random measure, generally defined on an extended probability space [Çinlar and Jacod (1981a, 1981b), Jacod and Protter (2011), Section 2.1.4]. If $\gamma>0$, we can thus represent the continuous local martingale component as $X_{t}^{\phi, c}=\int_{0}^{t} \sqrt{\gamma} \sigma\left(X_{s}^{\phi}\right) d \tilde{B}_{s}$, where 
$\tilde{B}$ is a standard Brownian motion (possibly defined on an extended probability space). The jump measure can be expressed in terms of a Poisson random measure. Such explicit representation is useful in applications for Monte Carlo simulation of Itô semimartingales as solutions of SDEs [Jacod and Protter (2011)]. Since our model arises as the time change, an alternative way to simulate it is by simulating the "background" process $(X, D)$ and the independent subordinator $\mathcal{T}$.

We now formulate Itô's formula for functions of the bi-variate process in the form convenient for our application. We first formulate Itô's formula for functions of $X^{\phi}$ only.

Theorem 3.4 (Itô's formula for $X^{\phi}$ ). Suppose $X^{\phi}$ starts from $X_{0}^{\phi}=$ $x>0$. For any function $f(t, x) \in C^{1,2}\left(\mathbb{R}_{+} \times(0, \infty)\right)$ if zero is an unattainable boundary for the process $X$ or $f(t, x) \in C^{1,2}\left(\mathbb{R}_{+} \times[0, \infty)\right)$ if zero is an attainable boundary for $X$, Itô's formula can be written in the following form:

$$
\begin{aligned}
f\left(t, X_{t}^{\phi}\right)= & f(0, x)+\int_{0}^{t}\left(\partial_{s}+\frac{1}{2} \gamma \sigma^{2}\left(X_{s}^{\phi}\right) \partial_{x}^{2}+b^{0, \phi}\left(X_{t}^{\phi}\right) \partial_{x}\right) f\left(s, X_{s}^{\phi}\right) d s \\
& +\int_{0}^{t} \int_{\mathbb{R}}\left(f\left(s, X_{s-}^{\phi}+y\right)-f\left(s, X_{s-}^{\phi}\right)-y \partial_{x} f\left(s, X_{s-}^{\phi}\right)\right) \\
& \times \mathbf{1}_{\{|y| \leq 1\}} \nu^{X^{\phi}}(d s, d y) \\
& +\int_{0}^{t} \int_{\mathbb{R}}\left(f\left(s, X_{s-}^{\phi}+y\right)-f\left(s, X_{s-}^{\phi}\right)\right) \mathbf{1}_{\{|y|>1\}} \mu^{X^{\phi}}(d s, d y) \\
& +\int_{0}^{t} \int_{\mathbb{R}}\left(f\left(s, X_{s-}^{\phi}+y\right)-f\left(s, X_{s-}^{\phi}\right)\right) \\
& \times \mathbf{1}_{\{|y| \leq 1\}}\left(\mu^{X^{\phi}}(d s, d y)-\nu^{X^{\phi}}(d s, d y)\right) \\
& +\int_{0}^{t} \partial_{x} f\left(s, X_{s}^{\phi}\right) d X_{s}^{\phi, c},
\end{aligned}
$$

where $\mu^{X^{\phi}}$ is the random measure associated to jumps of $X^{\phi}$, and $\nu^{X^{\phi}}$ is its compensator measure (3.22).

Proof. This form of Itô's formula based on characteristics can be found in Jacod and Protter (2011), equation (2.1.20), page 32.

This useful form of Itô's formula gives the canonical representation of the semimartingale $f\left(t, X_{t}^{\phi}\right)$ in terms of the predictable process of finite variation ("drift"), optional process of finite variation ("large jumps"), continuous 
local martingale component that is the stochastic integral with respect to $X^{\phi, c}$, and the purely discontinuous local martingale that is the stochastic integral with respect to the martingale random measure $\mu^{X^{\phi}}-\nu^{X^{\phi}}$ of compensated jumps of $X^{\phi}$ ("compensated small jumps"). We are now ready to present Itô's formula for the bi-variate process. Due to the decomposition

$$
f\left(t, X_{t}^{\phi}, D_{t}^{\phi}\right)=f_{1}\left(t, X_{t}^{\phi}\right)+\left(1-D_{t}^{\phi}\right)\left(f_{0}\left(t, X_{t}^{\phi}\right)-f_{1}\left(t, X_{t}^{\phi}\right)\right)
$$

and Theorem 3.4, it is sufficient to give Itô's formula for the product (1 $\left.D_{t}^{\phi}\right) f\left(t, X_{t}^{\phi}\right)$.

Theorem 3.5 [Itô's formula for $\left(X^{\phi}, D^{\phi}\right)$ ]. Suppose $\left(X^{\phi}, D^{\phi}\right)$ starts from $X_{0}^{\phi}=x>0$ and $D_{0}^{\phi}=d \in\{0,1\}$. For any function $f(t, x) \in C^{1,2}\left(\mathbb{R}_{+} \times\right.$ $(0, \infty))$ if zero is an unattainable boundary for the diffusion process $X$ or $f(t, x) \in C^{1,2}\left(\mathbb{R}_{+} \times[0, \infty)\right)$ if zero is an attainable boundary for $X$, we have

$$
\begin{aligned}
\left(1-D_{t}^{\phi}\right) f\left(t, X_{t}^{\phi}\right) & \\
= & (1-d) f(0, x) \\
& +\int_{0}^{t}\left(1-D_{s-}^{\phi}\right)\left(\partial_{s}+\frac{1}{2} \gamma \sigma^{2}\left(X_{s}^{\phi}\right) \partial_{x}^{2}+b^{1, \phi}\left(X_{t}^{\phi}\right) \partial_{x}-k^{\phi}\left(X_{s}^{\phi}\right)\right) f\left(s, X_{s}^{\phi}\right) d s \\
& +\int_{0}^{t} \int_{\mathbb{R}}\left(1-D_{s-}^{\phi}\right)\left(f\left(s, X_{s-}^{\phi}+y\right)-f\left(s, X_{s-}^{\phi}\right)-y \partial_{x} f\left(s, X_{s-}^{\phi}\right)\right) \\
(3.24) & \quad \times \mathbf{1}_{\{|y| \leq 1\}} \hat{\nu}(d s, d y) \\
& +\int_{0}^{t} \int_{\mathbb{R}}\left(1-D_{s-}^{\phi}\right)\left(f\left(s, X_{s-}^{\phi}+y\right)-f\left(s, X_{s-}^{\phi}\right)\right) \mathbf{1}_{\{|y|>1\}} \hat{\mu}(d s, d y) \\
& +\int_{0}^{t}\left(1-D_{s-}^{\phi}\right) \partial_{x} f\left(s, X_{s}^{\phi}\right) d X_{s}^{\phi, c}-\int_{0}^{t}\left(1-D_{s-}^{\phi}\right) f\left(s, X_{s-}^{\phi}\right) d M_{s}^{\phi} \\
& +\int_{0}^{t} \int_{\mathbb{R}}\left(1-D_{s-}^{\phi}\right)\left(f\left(s, X_{s-}^{\phi}+y\right)-f\left(s, X_{s-}^{\phi}\right)\right) \\
& \times \mathbf{1}_{\{|y| \leq 1\}}(\hat{\mu}(d s, d y)-\hat{\nu}(d s, d y)),
\end{aligned}
$$

where we introduced a random measure associated to those jumps of $X^{\phi}$ that do not coincide with jumps of $D^{\phi}$,

$$
\hat{\mu}(\omega ; d s, d y)=\sum_{u} \mathbf{1}_{\left\{\Delta X_{u}^{\phi}(\omega) \neq 0\right\}} \mathbf{1}_{\left\{\Delta D_{u}^{\phi}(\omega)=0\right\}} \delta_{\left(u, \Delta X_{u}^{\phi}(\omega)\right)}(d s, d y),
$$

and its compensator measure

$$
\begin{aligned}
& \hat{\nu}(\omega ; d s, d y) \\
& \quad=\left[\pi^{0, \phi}\left(X_{s-}^{\phi}, y\right)-\left(1-D_{s-}^{\phi}\right)\left(\pi^{0, \phi}\left(X_{s-}^{\phi}, y\right)-\pi^{1, \phi}\left(X_{s-}^{\phi}, y\right)\right)\right] d y d s .
\end{aligned}
$$


Proof. We start with Itô's product rule,

$$
\begin{aligned}
\left(1-D_{t}^{\phi}\right) f\left(t, X_{t}^{\phi}\right)= & (1-d) f(0, x)+\int_{0}^{t}\left(1-D_{s-}^{\phi}\right) d f\left(s, X_{s}^{\phi}\right) \\
& -\int_{0}^{t} f\left(s, X_{s-}^{\phi}\right) d D_{s}^{\phi}-\sum_{s \leq t} \Delta D_{s}^{\phi}\left(f\left(s, X_{s}^{\phi}\right)-f\left(s, X_{s-}^{\phi}\right)\right) .
\end{aligned}
$$

Due to Theorem 3.4, the second term is

$$
\begin{aligned}
\int_{0}^{t}(1- & \left.D_{s-}^{\phi}\right) d f\left(X_{s}^{\phi}\right) \\
= & \int_{0}^{t}\left(1-D_{s-}^{\phi}\right)\left(\partial_{s}+\frac{1}{2} \gamma \sigma^{2}\left(X_{s}^{\phi}\right) \partial_{x}^{2}+b^{0, \phi}\left(X_{t}^{\phi}\right) \partial_{x}\right) f\left(s, X_{s}^{\phi}\right) d s \\
& +\int_{0}^{t} \int_{\mathbb{R}}\left(1-D_{s-}^{\phi}\right)\left(f\left(s, X_{s-}^{\phi}+y\right)-f\left(s, X_{s-}^{\phi}\right)-y \partial_{x} f\left(s, X_{s-}^{\phi}\right)\right) \\
& \times \mathbf{1}_{\{|y| \leq 1\}} \nu^{X^{\phi}}(d s, d y) \\
& +\int_{0}^{t} \int_{\mathbb{R}}\left(1-D_{s-}^{\phi}\right)\left(f\left(s, X_{s-}^{\phi}+y\right)-f\left(s, X_{s-}^{\phi}\right)\right) \mathbf{1}_{\{|y|>1\}} \mu^{X^{\phi}}(d s, d y) \\
& +\int_{0}^{t} \int_{\mathbb{R}}\left(1-D_{s-}^{\phi}\right)\left(f\left(s, X_{s-}^{\phi}+y\right)-f\left(s, X_{s-}^{\phi}\right)\right) \\
& +\int_{0}^{t}\left(1-D_{\{|y| \leq 1\}}\left(\mu^{X^{\phi}}(d s, d y)-\partial_{x} f\left(s, X_{s}^{\phi}\right) d X_{s}^{\phi, c} .\right.\right.
\end{aligned}
$$

The third term is

$$
\begin{aligned}
\int_{0}^{t} f & \left(s, X_{s-}^{\phi}\right) d D_{s}^{\phi} \\
& =\int_{0}^{t}\left(1-D_{s-}^{\phi}\right) f\left(s, X_{s-}^{\phi}\right) d D_{s}^{\phi} \\
& =\int_{0}^{t}\left(1-D_{s-}^{\phi}\right) f\left(s, X_{s-}^{\phi}\right) d M_{s}^{\phi}+\int_{0}^{t}\left(1-D_{s-}^{\phi}\right) f\left(s, X_{s-}^{\phi}\right) k^{\phi}\left(X_{s-}^{\phi}\right) d s .
\end{aligned}
$$

The first equality is due to the fact that

$$
\int_{0}^{t} D_{s-}^{\phi} f\left(X_{s-}^{\phi}\right) d D_{s}^{\phi}=\sum_{s \leq t} D_{s-}^{\phi} f\left(X_{s-}^{\phi}\right) \Delta D_{s}^{\phi}=0,
$$

since $D_{s-}^{\phi} \Delta D_{s}^{\phi}=0$ (if $\Delta D_{s}^{\phi}=1$, then $D_{s-}^{\phi}=0$ ). In the second equality we used the Doob-Meyer decomposition of $D^{\phi}$. 
The fourth term is (in the first equality we again use $D_{s-}^{\phi} \Delta D_{s}^{\phi}=0$ )

$$
\begin{aligned}
\sum_{s \leq t} \Delta & D_{s}^{\phi}\left(f\left(X_{s}^{\phi}\right)-f\left(X_{s-}^{\phi}\right)\right) \\
= & \sum_{s \leq t}\left(1-D_{s-}^{\phi}\right) \Delta D_{s}^{\phi}\left(f\left(X_{s}^{\phi}\right)-f\left(X_{s-}^{\phi}\right)\right) \\
= & \int_{0}^{t} \int_{\mathbb{R}}\left(1-D_{s-}^{\phi}\right)\left(f\left(X_{s-}^{\phi}+y\right)-f\left(X_{s-}^{\phi}\right)\right) \\
& \times \mathbf{1}_{\{|y| \leq 1\}}(\tilde{\mu}(d s, d y)-\tilde{\nu}(d s, d y)) \\
& +\int_{0}^{t} \int_{\mathbb{R}}\left(1-D_{s-}^{\phi}\right)\left(f\left(X_{s-}^{\phi}+y\right)-f\left(X_{s-}^{\phi}\right)\right) \mathbf{1}_{\{|y|>1\}} \tilde{\mu}(d s, d y) \\
& +\int_{0}^{t} \int_{\mathbb{R}}\left(1-D_{s-}^{\phi}\right)\left(f\left(X_{s-}^{\phi}+y\right)-f\left(X_{s-}^{\phi}\right)-y \partial_{x} f\left(s, X_{s-}^{\phi}\right)\right) \\
& \quad \times \mathbf{1}_{\{|y| \leq 1\}} \tilde{\nu}(d s, d y) \\
& \quad \int_{0}^{t}\left(1-D_{s-}^{\phi}\right) \partial_{x} f\left(s, X_{s-}^{\phi}\right) \int_{\mathbb{R}} y \mathbf{1}_{\{|y| \leq 1\}} \tilde{\nu}(d s, d y),
\end{aligned}
$$

where we introduced a random measure associated to those jumps of $X^{\phi}$ that occur contemporaneously with jumps of $D^{\phi}$,

$$
\tilde{\mu}(\omega ; d s, d y)=\sum_{u} \mathbf{1}_{\left\{\Delta X_{u}^{\phi}(\omega) \neq 0\right\}} \mathbf{1}_{\left\{\Delta D_{u}^{\phi}(\omega)=1\right\}} \delta_{\left(u, \Delta X_{u}^{\phi}(\omega)\right)}(d s, d y),
$$

and its compensator measure

$$
\tilde{\nu}(\omega ; d s, d y)=\left(1-D_{s-}^{\phi}\right)\left(\pi^{0, \phi}\left(X_{s-}^{\phi}, y\right)-\pi^{1, \phi}\left(X_{s-}^{\phi}, y\right)\right) d y d s .
$$

To prove that this is the compensator of $\tilde{\mu}$, we note that for any Borel set $\mathcal{B} \in \mathbb{R} \backslash\{0\}$ the process

$$
\tilde{\mu}_{t}(\mathcal{B})(\omega):=\tilde{\mu}(\omega ;(0, t] \times \mathcal{B})
$$

is a one-point point process equal to one at time $t$ if $D_{t}^{\phi}-D_{0}^{\phi}=1$ (i.e., a jump of $D^{\phi}$ (default) occurs during the time interval $\left.(0, t]\right)$ and the process $X^{\phi}$ experiences a jump at the time of default $\tau$ with size in $\mathcal{B}, \Delta X_{\tau}^{\phi} \in \mathcal{B}$. The compensator of this process is readily computed from the compensator $\nu$ (3.21) of the measure $\mu$ associated to the jumps of $\left(X^{\phi}, D^{\phi}\right)$

$$
\begin{aligned}
\tilde{\nu}_{t}(\mathcal{B}) & =\tilde{\nu}((0, t] \times \mathcal{B}) \\
& =\int_{0}^{t} \int_{\mathcal{B} \times \mathbb{R}} y z \nu(d s, d y d z) \\
& =\int_{0}^{t} \int_{\mathcal{B}}\left(1-D_{s-}^{\phi}\right)\left(\pi^{0, \phi}\left(X_{s-}^{\phi}, y\right)-\pi^{1, \phi}\left(X_{s-}^{\phi}, y\right)\right) d y
\end{aligned}
$$


where the last equality follows by substituting equation (3.17) into equation (3.21) and doing the integration. Then $\left(\tilde{\mu}_{t}(\mathcal{B})-\tilde{\nu}_{t}(\mathcal{B})\right)+\tilde{\nu}_{t}(\mathcal{B})$ is the Doob-Meyer decomposition of $\tilde{\mu}_{t}(\mathcal{B})$.

We now put the pieces together and use the following identities to combine similar terms and arrive at the final result (3.24). First we observe that

$$
\mu^{X^{\phi}}=\hat{\mu}+\tilde{\mu} .
$$

This immediately follows from

$$
\begin{aligned}
\sum_{u} \mathbf{1}_{\left\{\Delta X_{u}^{\phi}(\omega) \neq 0\right\}} \delta_{\left(u, \Delta X_{u}^{\phi}(\omega)\right)}(d s, d y) & \\
= & \sum_{u} \mathbf{1}_{\left\{\Delta X_{u}^{\phi}(\omega) \neq 0\right\}} \mathbf{1}_{\left\{\Delta D_{u}^{\phi}(\omega)=0\right\}} \delta_{\left(u, \Delta X_{u}^{\phi}(\omega)\right)}(d s, d y) \\
& +\sum_{u} \mathbf{1}_{\left\{\Delta X_{u}^{\phi}(\omega) \neq 0\right\}} \mathbf{1}_{\left\{\Delta D_{u}^{\phi}(\omega)=1\right\}} \delta_{\left(u, \Delta X_{u}^{\phi}(\omega)\right)}(d s, d y)
\end{aligned}
$$

and accordingly for the compensators

$$
\nu^{X^{\phi}}=\hat{\nu}+\tilde{\nu}
$$

These identities allow us to combine integrals with the same integrands with respect to the random measures $\mu^{X^{\phi}}$ and $-\tilde{\mu}$ and $\nu^{X^{\phi}}$ and $-\tilde{\nu}$ into the ones with respect to $\hat{\mu}$ and $\hat{\nu}$.

Finally, we use the identity

$$
\begin{aligned}
\int_{\mathbb{R}} y \mathbf{1}_{\{|y| \leq 1\}} \tilde{\nu}(d s, d y) & \\
= & \left(1-D_{s-}^{\phi}\right) \int_{\mathbb{R}} y \mathbf{1}_{\{|y| \leq 1\}}\left(\pi^{0, \phi}\left(X_{s-}^{\phi}, y\right)-\pi^{1, \phi}\left(X_{s-}^{\phi}, y\right)\right) d y \\
= & \left(1-D_{s-}^{\phi}\right) \int_{(0, \infty)} \int_{\mathbb{R}} y \mathbf{1}_{\{|y| \leq 1\}}\left(p^{0}\left(u, X_{s-}^{\phi}, X_{s-}^{\phi}+y\right)\right. \\
& \left.-p^{1}\left(u, X_{s-}^{\phi}, X_{s-}^{\phi}+y\right)\right) d y \nu(d u) \\
& \left(1-D_{s-}^{\phi}\right)\left(b^{0, \phi}\left(X_{s-}^{\phi}\right)-b^{1, \phi}\left(X_{s-}^{\phi}\right)\right) d s
\end{aligned}
$$

to simplify the drift. The interchange of integrations in $u$ and $y$ is allowed due to the estimate (3.14) and the integrability properties of the Lévy measure, $\int_{(0, \infty)}(1 \wedge u) \nu(d u)<\infty$.

Itô's formula simplifies when the process $f\left(t, X_{t}^{\phi}, D_{t}^{\phi}\right)$ is a special semimartingale.

Corollary 3.3 [Itô's formula for $\left(X^{\phi}, D^{\phi}\right)$ - Special semimartingale version]. Suppose $\left(X^{\phi}, D^{\phi}\right)$ starts from $X_{0}^{\phi}=x>0$ and $D_{0}^{\phi}=d \in\{0,1\}$. For any function $f(t, x, d)=f_{1}(t, x)+(1-d)\left(f_{0}(t, x)+f_{1}(t, x)\right)$ with $f_{i}(t, x) \in$ 
$C^{1,2}\left(\mathbb{R}_{+} \times(0, \infty)\right)$ if zero is an unattainable boundary for the diffusion process $X$ or $f_{i}(t, x) \in C^{1,2}\left(\mathbb{R}_{+} \times[0, \infty)\right)$ if zero is an attainable boundary for $X$, if $f\left(t, X_{t}^{\phi}, D_{t}^{\phi}\right)$ is a special semimartingale [it suffices that either $X^{\phi}$ is a special semimartingale [i.e., $\int_{\mathbb{R}}\left(|y|^{2} \wedge|y|\right) \pi^{0, \phi}(x, y) d y<\infty$ for each $x \in I$ by Proposition 2.29 of Jacod and Shiryaev (2002), page 82] or the functions $f_{i}(t, x)$ are bounded], Itô's formula can be written in the following form:

$$
\begin{aligned}
f\left(t, X_{t}^{\phi}, D_{t}^{\phi}\right) & \\
= & f(0, x, d)-\int_{0}^{t}\left(1-D_{s-}^{\phi}\right)\left(f_{0}-f_{1}\right)\left(s, X_{s-}^{\phi}\right) d M_{s}^{\phi} \\
& +\int_{0}^{t}\left(\partial_{s}+\mathcal{A}^{\phi}\right) f\left(s, X_{s}^{\phi}, D_{t}^{\phi}\right) d s+\int_{0}^{t} \partial_{x} f\left(s, X_{s}^{\phi}, D_{t}^{\phi}\right) d X_{s}^{\phi, c} \\
& +\int_{0}^{t} \int_{\mathbb{R}}\left(f_{1}\left(s, X_{s-}^{\phi}+y\right)-f_{1}\left(s, X_{s-}^{\phi}\right)\right)\left(\mu^{X^{\phi}}(d s, d y)-\nu^{X^{\phi}}(d s, d y)\right) \\
& +\int_{0}^{t} \int_{\mathbb{R}}\left(\left(f_{0}-f_{1}\right)\left(s, X_{s-}^{\phi}+y\right)-\left(f_{0}-f_{1}\right)\left(s, X_{s-}^{\phi}\right)\right)\left(1-D_{s-}^{\phi}\right) \\
& \times(\hat{\mu}(d s, d y)-\hat{\nu}(d s, d y)),
\end{aligned}
$$

where $\mu^{X^{\phi}}$ is the random measure associated to jumps of $X^{\phi}$, and $\hat{\mu}$ is the random measure associated to those jumps of $X^{\phi}$ that do not coincide with jumps of $D^{\phi}$, and $\nu^{X^{\phi}}$ and $\hat{\nu}$ are their respective compensator measures (3.22) and (3.25). The generator $\mathcal{A}^{\phi}$ is given by equation (3.6).

Proof. The results follows immediately from Theorems 3.4 and 3.5, expression for the generator (3.6), and the canonical decomposition for the special semimartingale; cf. Proposition 2.29 of Jacod and Shiryaev (2002), page 82 .

This useful version of Itô's formula gives a canonical decomposition of the special semimartingale $f\left(t, X_{t}^{\phi}, D_{t}^{\phi}\right)$ into the predictable process of finite variation (explicitly given in terms of the generator $\mathcal{A}^{\phi}$ in the Markovian case considered here), a continuous local martingale part and a purely discontinuous local martingale part. The general form of it can be found in Theorem 3.89 of Jacod (1979), page 109.

Next we show the following useful sufficient condition for the "specialness" of the subordinate diffusion $X^{\phi}$.

Theorem 3.6 (Condition for specialness of $X^{\phi}$ ). If the diffusion $X$ has a stationary density

$$
\lim _{t \rightarrow \infty} p^{0}(t, x, y):=\pi(y)
$$


with the finite first moment $\int_{I} y \pi(y) d y<\infty$, then the subordinate diffusion $X_{t}^{\phi}$ is a special semimartingale.

Proof. By Proposition 2.29 of Jacod and Shiryaev (2002), page 82, and given our previous results, it suffices to show that $\int_{\{|y|>1\}}|y| \pi^{0, \phi}(x, y) d y<\infty$ for each $x \in I$. From McKean (1956) [see also Borodin and Salminen (2002), page 13], under our assumptions the transition density $p^{0}(t, x, y)$ can be written in the form $p^{0}(t, x, y)=m(y) p_{m}^{0}(t, x, y)$, where $m$ is the speed density of the diffusion $X$ given by

$$
m(x)=\frac{2}{\sigma^{2}(x) s(x)}, \quad s(x)=\exp \left\{-\int_{x_{0}}^{x} \frac{2 b(y)}{\sigma^{2}(y)} d y\right\}
$$

where $x_{0}>0$ in the definition of the scale density $s(x)$ is an arbitrary point [see Borodin and Salminen (2002) for the definitions of the scale function and the speed measure of a one-dimensional diffusion; under our assumptions the scale function and the speed measure of $X$ are absolutely continuous with respect to the Lebesgue measure with the densities given by equation (3.26)], and $p_{m}^{0}(t, x, y)=p_{m}^{0}(t, y, x)$ is symmetric and jointly continuous in $t, x, y$. The diffusion $X$ admits a stationary density if and only if the speed density is integrable on $I$ and, in this case, $\pi(x)=m(x) / \int_{I} m(y) d y$ [cf. Borodin and Salminen (2002), page 20]. In this case we can write the Lévy density of $X^{\phi}$ as

$$
\pi^{0, \phi}(x, y)=\pi(x+y) \int_{(0, \infty)} p_{m}^{0}(s, x, x+y) \nu(d s)
$$

for all $y \neq 0$, where we chose $x_{0}$ in the definition of speed density so that $\int_{I} m(y) d y=1$ and $\pi(x)=m(x)$. Since the function $\int_{(0, \infty)} p_{m}^{0}(s, x, x+y) \nu(d s)$ is bounded on the set $\{|y|>1\}, \int_{\{|y|>1\}}|y| \pi^{0, \phi}(x, y) d y<\infty$ follows immediately from the assumption $\int_{I} y \pi(y) d y<\infty$.

We note that many diffusions $X$ used in default intensity models, such as the CIR, the $3 / 2$, and the quadratic models given in the examples in Section 2, have stationary densities, so that the resulting time changed processes $X^{\phi}$ turn out to be special semimartingales. The canonical decomposition of the special semimartingale $X^{\phi}$ can then be written in the following form:

$$
X_{t}^{\phi}=x+A_{t}^{X^{\phi}}+X_{t}^{\phi, c}+\int_{0}^{t} \int_{\mathbb{R}} y\left(\mu^{X^{\phi}}(d s, d y)-\pi^{0, \phi}\left(X_{t-}^{\phi}, y\right) d y d s\right)
$$

with the predictable finite variation part

$$
A_{t}^{X^{\phi}}=\int_{0}^{t}\left(\gamma b\left(X_{t}^{\phi}\right)+\int_{(0, \infty)}\left(\int_{\mathbb{R}} y p^{0}\left(u, X_{t}^{\phi}, X_{t}^{\phi}+y\right) d y\right) \nu(d u)\right) d s
$$


with respect to the truncation function $h^{X^{\phi}}(x)=x$ [note that it differs from (3.20) with respect to the truncation function $h^{X^{\phi}}(x)=x \mathbf{1}_{\{|x| \leq 1\}}$ ], the continuous local martingale part that can be represented as $X_{t}^{\phi, c}=$ $\int_{0}^{t} \sqrt{\gamma} \sigma\left(X_{s}^{\phi}\right) d \tilde{B}_{s}$ and the purely discontinuous local martingale with jumps with the compensator $\pi^{0, \phi}\left(X_{t-}^{\phi}, y\right) d y d s$. From Example 2.2, we observe that the CIR diffusion satisfies the conditions of Theorem 3.6 for all $\kappa, \theta, \sigma>0$ without any further conditions on the coefficients.

4. Pricing credit-sensitive securities. We now discuss applications to the pricing of credit-sensitive securities. We make the usual assumptions of frictionless arbitrage-free markets, assume that the probability measure we are working with is an equivalent martingale measure chosen by the market, and that, under this probability measure, the default time $\tau$ of the obligor is modeled by the jump time of the process $D^{\phi}$, that is, $\tau=\inf \left\{t \geq 0: D_{t}^{\phi}=1\right\}$ (the case of $D_{0}^{\phi}=1$ and, hence, $\tau=0$, corresponds to the case when the obligor is already in default at time zero). Thus, the bivariate process $\left(X^{\phi}, D^{\phi}\right)$ under the EMM describes all the financial information in our model relevant for the risk-neutral pricing of credit-sensitive securities. We remark that our model falls into the general framework of default times of Janson, M'Baye and Protter (2011), with the underlying information flow affecting default generated by a Markovian Itô semimartingale and with the compensator of the default indicator $D^{\phi}$ absolutely continuous with respect to Lebesgue measure with intensity $\left(1-D_{s}^{\phi}\right) k^{\phi}\left(X_{t}^{\phi}\right)$, which, in our case, is explicitly computed via the application of the Phillips Theorem 3.1.

Consider a security with a promised payment $f_{0}\left(X_{T}^{\phi}\right)$ at maturity $T>0$ if default does not occur by time $T$ and a "recovery" payment $f_{1}\left(X_{T}^{\phi}\right)$ at maturity if default occurs. We generally allow the promised payment to depend on the state variable at maturity. This is the case when pricing options on credit spreads, where the credit spread at option's maturity is the function of the credit state variable at that time. This is also the case when pricing equity options in unified credit-equity models, where the state variable also drives the stock price observable up to the time of default. Depending on the context of the model, the recovery payment at maturity can be either taken constant, $f_{1}(x)=R$, if we do not assume that the state variable $X_{T}^{\phi}$ is observable to the investor after default, or taken to be a function of the state variable at maturity if the context of the model allows the investor to observe the state variable after default. In some applications, where the state variable drives the credit spread prior to default or in the credit-equity modeling framework, where the state variable drives the stock price prior to default, the recovery at maturity is taken to be constant. On the other hand, if one considers the framework where the firm defaults at 
time $\tau$ on its liabilities but continues to operate through the reorganization process (such as Chapter 11), and the final recovery settlement of the claims is made based on the outcome of restructuring, then in such applications it may make sense to model recovery as a function of the state variable at the time of payment. Our mathematical framework can accommodate both types of applications.

Thus, securities we consider are defined by payoff functions $f(x, d)$ with decomposition (2.4), where $f_{0}(x)$ is interpreted as the promised payment if no default occurs by maturity and $f_{1}(x)$ as the recovery paid at maturity if default occurs. The defaultable zero-coupon bond with unit face value is the simplest such security with $f_{0}=1$ and constant recovery $f_{1}=R \in[0,1]$. The security pricing in this model follows from the general results of the previous section. The payoff we consider is

$$
f\left(X_{T}^{\phi}, D_{T}^{\phi}\right)=f_{1}\left(X_{T}^{\phi}\right)-\left(1-D_{T}^{\phi}\right)\left(f_{1}\left(X_{T}^{\phi}\right)-f_{0}\left(X_{T}^{\phi}\right)\right),
$$

at time $T$. The price process of the security with this payoff is

$$
\begin{aligned}
f(t, & \left.X_{t}^{\phi}, D_{t}^{\phi}\right) \\
& =e^{-r(T-t)} \mathbb{E}\left[f\left(X_{T}^{\phi}, D_{T}^{\phi}\right) \mid \mathcal{H}_{t}^{\phi}\right] \\
& =e^{-r(T-t)} \mathcal{P}_{T-t}^{0, \phi} f_{1}\left(X_{t}^{\phi}\right)+\left(1-D_{t}^{\phi}\right) e^{-r(T-t)} \mathcal{P}_{T-t}^{1, \phi}\left(f_{0}-f_{1}\right)\left(X_{t}^{\phi}\right),
\end{aligned}
$$

where $r \geq 0$ is the risk-free interest rate assumed constant (but see Remark 4.1 at the end of this section). In particular, the price process of the defaultable zero-coupon bond with unit face value $f_{0}=1$ and zero recovery $f_{1}=0$ in the event of default is

$$
\mathcal{Z}\left(t, X_{t}^{\phi}, D_{t}^{\phi} ; T\right)=e^{-r(T-t)} \mathcal{Q}\left(t, X_{t}^{\phi}, D_{t}^{\phi} ; T\right),
$$

where $\mathcal{Q}\left(t, X_{t}^{\phi}, D_{t}^{\phi} ; T\right)$ is the survival probability to survive up to time $T$, given the state at time $t$,

$$
\mathcal{Q}\left(t, X_{t}^{\phi}, D_{t}^{\phi} ; T\right)=\mathbb{E}\left[\left(1-D_{T}^{\phi}\right) \mid \mathcal{H}_{t}^{\phi}\right]=\left(1-D_{t}^{\phi}\right) P_{T-t}^{1, \phi}\left(X_{t}^{\phi}, I\right),
$$

where $P_{t}^{1, \phi}(x, I)=\mathcal{P}_{t}^{1, \phi} 1(x)$. The term structure of credit spreads for defaultable bonds of all maturities as observed at time $t$, given the state $X_{t}^{\phi}$ and $D_{t}^{\phi}=0$, is

$$
\mathcal{S}\left(t, X_{t}^{\phi} ; T\right)=-\frac{1}{(T-t)} \ln \mathcal{P}_{T-t}^{1, \phi} 1\left(X_{t}^{\phi}\right) .
$$

In those applications where the recovery at maturity is assumed constant, $f_{1}=R$, the pricing formula simplifies to

$$
\begin{aligned}
f\left(t, X_{t}^{\phi}, D_{t}^{\phi}\right)= & e^{-r(T-t)}\left(1-D_{t}^{\phi}\right) \mathcal{P}_{T-t}^{1, \phi} f_{0}\left(X_{t}^{\phi}\right) \\
& +e^{-r(T-t)} R\left(1-\mathcal{Q}\left(t, X_{t}^{\phi}, D_{t}^{\phi} ; T\right)\right),
\end{aligned}
$$


and the investor who observes the price processes of traded securities in this market can determine whether or not default has occurred, as well as can filter out the state variable $X^{\phi}$ prior to default $\tau$ from the prices of traded securities. In this case, when the recovery payment is not allowed to depend on $X_{T}^{\phi}$ (assumed unobservable in such applications), the investor's filtration is smaller than the filtration generated by $\left(X_{t}^{\phi}, D_{t}^{\phi}\right)$ since $X_{t}^{\phi}$ is only observed by the investor prior to default time $\tau$. In fact, in such applications the investor's filtration can be identified with the filtration generated by $\left(Y_{t}^{\phi}, D_{t}^{\phi}\right)$, where the process $Y_{t}^{\phi}:=\left(1-D_{t}^{\phi}\right) X_{t}^{\phi}$ jumps to zero at default and stays there. Applying Itô's formula in the form of Theorem 3.5, this semimartingale has the canonical representation $\left[Y_{0}^{\phi}=\left(1-D_{0}^{\phi}\right) X_{0}^{\phi}\right]$

$$
\begin{aligned}
Y_{t}^{\phi}= & Y_{0}^{\phi}+\int_{0}^{t}\left(1-D_{s-}^{\phi}\right)\left(b^{1, \phi}\left(Y_{s-}^{\phi}\right)-k^{\phi}\left(Y_{s-}^{\phi}\right) Y_{s-}^{\phi}\right) d s \\
& +\int_{0}^{t} \int_{\mathbb{R}} y \mathbf{1}_{\{|y|>1\}}\left(1-D_{s-}^{\phi}\right) \hat{\mu}(d s d y) \\
& +\int_{0}^{t} \int_{\mathbb{R}} y \mathbf{1}_{\{|y| \leq 1\}}\left(1-D_{s-}^{\phi}\right)\left(\hat{\mu}(d s d y)-\pi^{1, \phi}\left(Y_{s-}^{\phi}, y\right) d y d s\right) \\
& +\int_{0}^{t}\left(1-D_{s-}^{\phi}\right) d X_{s}^{\phi, c}-\int_{0}^{t} Y_{s-}^{\phi} d M_{s}^{\phi} .
\end{aligned}
$$

This canonical representation decomposes $Y^{\phi}$ into the "drift," "large jumps" prior to default, a purely discontinuous local martingale of "small jumps" prior to default with the compensator measure $\left(1-D_{s-}^{\phi}\right) \pi^{1, \phi}\left(X_{s-}^{\phi}, y\right) d y d s$ [observe from equation $(3.25)$ that $\left(1-D_{s-}^{\phi}\right) \hat{\nu}(d s, d y)=\left(1-D_{s-}^{\phi}\right) \pi^{1, \phi}\left(X_{s-}^{\phi}\right.$, $y) d y d s]$, a continuous local martingale component that can be further represented as $\int_{0}^{t}\left(1-D_{s-}^{\phi}\right) \sqrt{\gamma} \sigma\left(Y_{s-}^{\phi}\right) d \tilde{B}_{s}$ in terms of a Brownian motion, and a final jump to zero (the default term $-\int_{0}^{t} Y_{s}^{\phi} d M_{s}^{\phi}$ ). In the credit-equity context, one identifies the process $Y_{t}^{\phi}$ with the defaultable stock price process; see, for example, Mendoza-Arriaga, Carr and Linetsky (2010) and MendozaArriaga and Linetsky (2013) for the multi-firm case. We further remark that Lorig, Lozano-Carbassé and Mendoza-Arriaga (2013) apply the canonical representation (4.6) of the stock price process $Y_{t}^{\phi}$ to the valuation of variance swaps on individual stocks with the risk of bankruptcy.

So far we have considered recovery payments at maturity. Recovery at the time of default can also be treated in our framework. Suppose that if default occurs prior to maturity $T$, the recovery is received by the investor at the time of default $\tau$ and is equal to some function of the state variable $X_{\tau}^{\phi}$ at the time of default, $\mathcal{R}\left(X_{\tau}^{\phi}\right)$. By the standard calculation in credit risk modeling [cf. Lemma 7.3.4.3(i) in Jeanblanc, Yor and Chesney (2009), 
page 421], the value of such recovery at time $t$ prior to maturity $T$ is then given by

$$
\begin{aligned}
\mathbb{E}\left[e^{-r(\tau-t)} \mathcal{R}\left(X_{\tau}^{\phi}\right) \mid \mathcal{H}_{t}^{\phi}\right]= & \left(1-D_{t}^{\phi}\right) \int_{t}^{T} e^{-r(u-t)} \mathcal{P}_{u-t}^{1, \phi}\left(\mathcal{R} \cdot k^{\phi}\right)\left(X_{t}^{\phi}\right) d u \\
& +e^{r(t-\tau)} \mathcal{R}\left(X_{\tau}^{\phi}\right) D_{t}^{\phi},
\end{aligned}
$$

where $\left(\mathcal{R} \cdot k^{\phi}\right)(x)=\mathcal{R}(x) k^{\phi}(x)$.

REMARK 4.1 (Risk-free interest rates). We remark that stochastic riskfree interest rates can be handled in our subordinate diffusion framework as follows. The subordinate semigroup $\left(\mathcal{P}_{t}^{1, \phi}\right)_{t \geq 0}$ is taken to be the pricing semigroup. Namely, the state variable $Z^{\phi}$ driving the term structure of interest rates is assumed to be a Markovian Itô semimartingale with the following dynamics under the equivalent martingale measure:

$$
\begin{aligned}
Z_{t}^{\phi}= & Z_{0}^{\phi}+\int_{0}^{t} b^{1, \phi}\left(Z_{s-}^{\phi}\right) d s+\int_{0}^{t} \int_{\mathbb{R}} y \mathbf{1}_{\{|y|>1\}} \mu^{Z^{\phi}}(d s, d y)+Z_{t}^{\phi, c} \\
& +\int_{0}^{t} \int_{\mathbb{R}} y \mathbf{1}_{\{|y| \leq 1\}}\left(\mu^{Z^{\phi}}(d s, d y)-\pi^{1, \phi}\left(Z_{s-}^{\phi}, y\right) d y d s\right),
\end{aligned}
$$

where $Z_{t}^{\phi, c}=\int_{0}^{t} \sqrt{\gamma} \sigma\left(Z_{s}^{\phi}\right) d \tilde{B}_{s}$ with a standard Brownian motion $\tilde{B}$. The random measure $\mu^{Z^{\phi}}$ on $\mathbb{R}_{+} \times(\mathbb{R} \backslash\{0\})$ associated to jumps of $Z^{\phi}$ has a compensator $\nu^{Z^{\phi}}(d s, d y)=\pi^{1, \phi}\left(Z_{s-}^{\phi}, y\right) d y d s$ with $\pi^{1, \phi}(x, y)$ given by equation (3.8) with $\beta=1$. The function $b^{1, \phi}(x)$ in the drift is given by equation (3.9) with $\beta=1$. Similarly to Theorem 3.6, it is easy to show the following.

Proposition 4.1 (Condition for specialness of $Z^{\phi}$ ). If

$$
\lim _{t \rightarrow \infty} p^{0}(t, x, y):=\pi(y)
$$

with the finite first moment $\int_{I} y \pi(y) d y<\infty$, then $Z^{\phi}$ is a special semimartingale.

Proof. Recall that for the density of the Feynman-Kac semigroup $\mathcal{P}^{1}$ we have [cf. Revuz and Yor (1999), page 358]

$$
p^{1}(t, x, y)=\mathbb{E}_{x}\left[e^{-\int_{0}^{t} k\left(X_{u}\right) d u} \mid X_{t}=y\right] p^{0}(t, x, y) \leq p^{0}(t, x, y)
$$

for each $x, y \in I$ and $t>0$. Under our assumptions, this implies that $\int_{\mathbb{R}}\left(|y|^{2} \wedge\right.$ $|y|) \pi^{1, \phi}(x, y) d y \leq \int_{\mathbb{R}}\left(|y|^{2} \wedge|y|\right) \pi^{0, \phi}(x, y) d y<\infty$, where the second inequality follows from the proof of Theorem 3.6. Thus $Z^{\phi}$ is special by Proposition 2.29 of Jacod and Shiryaev (2002), page 82. 
In the case of special $Z^{\phi}$, the canonical decomposition of $Z^{\phi}$ reads

$$
Z_{t}^{\phi}=x+A_{t}^{Z^{\phi}}+Z_{t}^{\phi, c}+\int_{0}^{t} \int_{\mathbb{R}} y\left(\mu^{Z^{\phi}}(d s, d y)-\pi^{1, \phi}\left(Z_{t-}^{\phi}, y\right) d y d s\right)
$$

with the predictable finite variation part

$$
A_{t}^{Z^{\phi}}=\int_{0}^{t}\left(\gamma b\left(Z_{t}^{\phi}\right)+\int_{(0, \infty)}\left(\int_{\mathbb{R}} y p^{1}\left(u, Z_{t}^{\phi}, Z_{t}^{\phi}+y\right) d y\right) \nu(d u)\right) d s
$$

with respect to the truncation function $h^{X^{\phi}}(x)=x$, the continuous local martingale part that can be represented as $Z_{t}^{\phi, c}=\int_{0}^{t} \sqrt{\gamma} \sigma\left(Z_{s}^{\phi}\right) d \tilde{B}_{s}$ and the purely discontinuous local martingale with jumps with the compensator $\pi^{1, \phi}\left(Z_{t-}^{\phi}, y\right) d y d s$. Most popular short-rate diffusions, such as CIR, 3/2, etc., have stationary densities due to mean-reversion. By Proposition 4.1, the corresponding subordinate short-rate models are driven by jump-diffusion or pure jump processes $Z^{\phi}$ that are special semimartingales. The short rate process is taken to be

$$
r_{t}=k^{\phi}\left(Z_{t}^{\phi}\right)
$$

where $k^{\phi}(x)$ is given by equation (3.10), and the money market account is $A_{t}=e^{\int_{0}^{t} r_{s} d s}=e^{\int_{0}^{t} k^{\phi}\left(Z_{s}^{\phi}\right) d s}$. The pricing semigroup is then the semigroup $\left(\mathcal{P}_{t}^{1, \phi}\right)_{t>0}$ with generator $\mathcal{A}^{1, \phi}$, the subordinate semigroup of the FeynmanKac semigroup $\left(\mathcal{P}_{t}^{1}\right)_{t \geq 0}$ with generator $\mathcal{A}^{1}$ and, in particular, for the risk-free zero-coupon bond we have

$$
P\left(Z_{t}^{\phi}, t ; T\right)=\mathcal{P}_{T-t}^{1, \phi} 1\left(Z_{t}^{\phi}\right)=P_{T-t}^{1, \phi}\left(Z_{t}^{\phi}, I\right) .
$$

Now an extension to the combined model that includes both the subordinate diffusion risk-free interest rate model and the subordinate default intensity model is immediate, as long as the interest rate model and the default model are assumed independent. At the expense of increased complexity, dependence can be further introduced either by starting with independent factors, each following a subordinate diffusion, and then combining them in a multi-dimensional model by taking linear combinations of independent factors, or by means of multivariate subordination as in Mendoza-Arriaga and Linetsky (2013).

5. Eigenfunction expansions of subordinate semigroups. We now show how to explicitly compute the semigroups $\left(\mathcal{P}_{t}^{\beta, \phi}\right)_{t \geq 0}$ by the eigenfunction expansion method. We start by observing that for any $f \in C_{c}^{2}(I)$ the infinitesimal generator $\mathcal{A}^{\beta}$ of $\left(\mathcal{P}_{t}^{\beta}\right)_{t \geq 0}$ can be re-written in the formally self-adjoint form using the scale and speed densities (3.26)

$$
\mathcal{A}^{\beta} f(x)=\frac{1}{m(x)}\left(\frac{f^{\prime}(x)}{s(x)}\right)^{\prime}-\beta k(x) f(x) .
$$


Indeed, $\mathcal{A}^{\beta}$ can be extended to a self-adjoint operator in the Hilbert space $L^{2}(I, m)$ of functions on $I$ square-integrable with the speed measure $m(d x)=$ $m(x) d x$ and endowed with the inner product

$$
(f, g)=\int_{I} f(x) g(x) m(x) d x .
$$

Furthermore, the restriction of $\left(\mathcal{P}_{t}^{\beta}\right)_{t \geq 0}$ to $C([0, \infty]) \cap L^{2}(I, m)$ can then be extended to a strongly-continuous semigroup of symmetric contractions in the Hilbert space $L^{2}(I, m)$. Thus, the spectral theorem for self-adjoint operators in Hilbert space can be applied to write down the spectral decomposition of $\mathcal{A}^{\beta}$ and $\left(\mathcal{P}_{t}^{\beta}\right)_{t \geq 0}$. The spectral representation for one-dimensional diffusions goes back to the classical work of McKean (1956) [see also Itô and McKean (1974), Section 4.11]. More generally, one-dimensional diffusions are examples of symmetric Markov processes whose transition semigroups admit symmetric extensions to the Hilbert space $L^{2}(E, m)$, where $E$ is the state space of the Markov process, and $m$ is a positive Radon measure on $E$ with full support. Fukushima, Oshima and Takeda (2011) and Chen and Fukushima (2011) are the standard references on the subject. In the case of one-dimensional diffusions, $E=I$ is the interval on the real line, and $m$ is the speed measure. An excellent exposition of the spectral theorem and applications to subordination can be found in Schilling, Song and Vondraček (2010), Chapters 10 and 11. Surveys of applications of the spectral expansion method to diffusion models in finance can be found in Li and Linetsky (2004, 2008), where an extensive bibliography is given. Recent applications of subordinate diffusion models in finance can be found in Boyarchenko and Levendorskiǔ (2007), Mendoza-Arriaga, Carr and Linetsky (2010), Li and Linetsky (2013a, 2013b), Mendoza-Arriaga and Linetsky (2013), Lim, Li and Linetsky (2012). Here we give a brief account limited to needs of the present paper.

For computational simplicity here we limit ourselves to the special case when the diffusion $X$ and the function $k$ are such that $\left(\mathcal{P}_{t}^{\beta}\right)_{t \geq 0}$ in $L^{2}(I, m)$ are trace-class semigroups for $\beta \geq 0$, that is, the operators $\mathcal{P}_{t}^{\beta}$ are traceclass for all $t>0$ and $\beta \geq 0$. Recall that for a positive semi-definite operator $A$ on a separable Hilbert space $\mathcal{H}$, the trace of $A$ is defined by $\operatorname{tr} A=\sum_{n=1}^{\infty}\left(\varphi_{n}, A \varphi_{n}\right) \in[0, \infty]$, where $\varphi_{n}$ is some orthonormal basis in $\mathcal{H}$. The trace is independent of the orthonormal basis chosen; cf. Reed and Simon (1980), page 206. A positive semi-definite operator is called trace-class if and only if its trace is finite. The semigroup operators $\mathcal{P}_{t}^{\beta}$ are positive semi-definite. Under the assumption that $\mathcal{P}_{t}^{\beta}$ are trace-class for all $t>0$, the spectra of each $\mathcal{P}_{t}^{\beta}$, as well as of the generators $\mathcal{A}^{\beta}$ of the semigroups $\left(\mathcal{P}_{t}^{\beta}\right)_{t \geq 0}$ in $L^{2}(I, m)$, are purely discrete with eigenvalues $\left(e^{-\lambda_{n}^{\beta} t}\right)_{n \geq 1}$ (for $\left.t>0\right)$ and 
$\left(-\lambda_{n}^{\beta}\right)_{n \geq 1}$ respectively, and

$$
\operatorname{tr} \mathcal{P}_{t}^{\beta}=\sum_{n=1}^{\infty} e^{-\lambda_{n}^{\beta} t}<\infty
$$

for all $t>0$; cf. Lemma 7.2.1 of Davies (2007). Here $0 \leq \lambda_{1}^{\beta} \leq \lambda_{2}^{\beta} \leq \cdots$ are arranged in increasing order and repeated according to multiplicity. Then the function $\mathcal{P}_{t}^{\beta} f(x)$ has an eigenfunction expansion of the form

$$
\mathcal{P}_{t}^{\beta} f(x)=\sum_{n=1}^{\infty} f_{n}^{\beta} e^{-\lambda_{n}^{\beta} t} \varphi_{n}^{\beta}(x), \quad f_{n}^{\beta}=\left(f, \varphi_{n}^{\beta}\right)
$$

for any $f \in L^{2}(I, m)$ and all $t \geq 0$,

where $\varphi_{n}^{\beta}$ is the $n$ th-eigenfunction

$$
\mathcal{P}_{t}^{\beta} \varphi_{n}^{\beta}=e^{-\lambda_{n}^{\beta} t} \varphi_{n}^{\beta} \quad \text { and } \quad \mathcal{A}^{\beta} \varphi_{n}=-\lambda_{n}^{\beta} \varphi_{n}^{\beta} .
$$

The eigenfunctions $\left(\varphi_{n}^{\beta}\right)_{n \geq 1}$ form a complete orthonormal basis in $L^{2}(I, m)$, and $f_{n}^{\beta}$ is the $n$th expansion coefficient in this basis.

For a trace-class semigroup, each $\mathcal{P}_{t}^{\beta}$ with $t>0$ admits a symmetric kernel $p_{m}^{\beta}(t, x, y) \in L^{2}(I \times I, m \times m)$ with respect to the measure $m$ [i.e., $p_{m}^{\beta}(t, x, y)=p_{m}^{\beta}(t, y, x), \mathcal{P}_{t}^{\beta} f(x)=\int_{I} p_{m}^{\beta}(t, x, y) f(y) m(d y)$ for $f \in L^{2}(I, m)$, and $\left.\int_{I \times I}\left(p_{m}^{\beta}(t, x, y)\right)^{2} m(d x) m(d y)<\infty\right]$, which has the following bi-linear expansion:

$$
p_{m}^{\beta}(t, x, y)=\sum_{n=1}^{\infty} e^{-\lambda_{n}^{\beta} t} \varphi_{n}^{\beta}(x) \varphi_{n}^{\beta}(y) .
$$

The expansions in (5.2) and (5.4) in general converge under the $L^{2}(I, m)$ and $L^{2}(I \times I, m \times m)$ norms, respectively. Moreover, since for one-dimensional diffusions for each $t>0$ the kernel $p_{m}^{\beta}(t, x, y)$ with respect to the speed measure is jointly continuous in $x$ and $y$ (and $t$ ) by the results of McKean (1956), then each eigenfunction $\varphi_{n}^{\beta}$ is continuous, and satisfies the estimate

$$
\left|\varphi_{n}^{\beta}(x)\right| \leq e^{\lambda_{n}^{\beta} t / 2} \sqrt{p_{m}^{\beta}(t, x, x)}
$$

for all $n, x$ and $t>0$. Moreover, for any $f \in L^{2}(I, m)$, expansion (5.2) converges uniformly in $x$ on compacts for each $t>0$ to the function $\mathcal{P}_{t}^{\beta} f(x)$ continuous in $x$, and the bi-linear expansion (5.4) converges uniformly on compacts; cf. Theorem 7.2.5 of Davies (2007).

The spectral representation for the density of a 1D diffusion with respect to the speed measure was obtained by McKean (1956); see also Itô and McKean (1974), Section 4.11. In general, the spectrum contains some continuous 
spectrum, and the spectral representation is in terms of the integral with respect to the spectral measure. Nevertheless, many diffusions arising in finance applications have purely discrete spectra with explicitly known eigenfunctions and eigenvalues satisfying the trace class condition (5.1) for all $t>0$, including OU, CIR, CEV and JDCEV diffusions; see surveys Linetsky $(2004,2008)$ and references therein for finance applications.

We now summarize key results about the eigenfunction expansion of the subordinate semigroups $\left(\mathcal{P}_{t}^{\beta, \phi}\right)_{t \geq 0}$ defined in Section 3 .

TheOrem 5.1. Suppose the semigroup $\left(\mathcal{P}_{t}^{\beta}\right)_{t>0}$ defined in Section 2 is trace-class with eigenvalues and eigenfunctions $e^{-\lambda_{n}^{\beta} t}$ and $\varphi_{n}^{\beta}(x)$, respectively. Further suppose that the eigenfunctions have bounds

$$
\left|\varphi_{n}^{\beta}(x)\right| \leq C_{K}^{\beta}
$$

on each compact set $K \subset I$ with $C_{K}^{\beta}$ independent of $n$ but possibly dependent on $K$. Let $\mathcal{T}$ be a subordinator with the Laplace exponent satisfying the following condition for all $t>0$ :

$$
\sum_{n=1}^{\infty} e^{-\phi\left(\lambda_{n}^{\beta}\right) t}<\infty
$$

Then the subordinate semigroup $\left(\mathcal{P}_{t}^{\beta, \phi}\right)_{t \geq 0}$ is a strongly continuous semigroup of symmetric contractions on $L^{2}(I, m)$, trace-class for all $t>0$ with the eigenvalues $e^{-\phi\left(\lambda_{n}^{\beta}\right) t}$ and normalized eigenfunctions $\varphi_{n}^{\beta}(x)$, and possesses a continuous in $x, y$ density with respect to the speed measure $m(d x)$ that is given by the bi-linear expansion

$$
p_{m}^{\beta, \phi}(t, x, y)=\sum_{n=0}^{\infty} e^{-\phi\left(\lambda_{n}^{\beta}\right) t} \varphi_{n}^{\beta}(x) \varphi_{n}^{\beta}(y)
$$

uniformly convergent in $x, y$ on compacts in $I \times I$ for all $t>0$. For each $f \in$ $L^{2}(I, m)$ and $t>0$ the function $\mathcal{P}_{t}^{\beta, \phi} f(x)$ has the eigenfunction expansion

$$
\mathcal{P}_{t}^{\beta, \phi} f(x)=\sum_{n=1}^{\infty} e^{-\phi\left(\lambda_{n}^{\beta}\right) t} f_{n}^{\beta} \varphi_{n}^{\beta}(x), \quad f_{n}^{\beta}=\left(f, \varphi_{n}^{\beta}\right)
$$

uniformly convergent in $x$ on compacts in $I$.

Without bound (5.5) on the eigenfunctions $\varphi_{n}^{\beta}(x)$ and the trace-class condition (5.6) on the Laplace exponent of the subordinator, the eigenfunction expansions (5.7)-(5.8) generally converge in $L^{2}(I \times I, m \times m)$ and $L^{2}(I, m)$, respectively, but not necessarily uniformly. The bound on eigenfunctions and the trace-class condition on the subordinator are sufficient to ensure 
uniform convergence. The bound on eigenfunctions is satisfied for many diffusions important in finance applications, such as OU, CIR, CEV, JDCEV and models related to these diffusions. Condition (5.6) also turns out to be mild and is satisfied in many applications in finance. For example, it is satisfied for tempered stable subordinators of Example 3.1 with $\alpha \in(0,1)$ when eigenvalues grow linearly in the eigenvalue number, as is the case for OU, CIR, CEV and JDCEV diffusions. The key observation of practical importance is that, in the context of the eigenfunction expansion method, subordination simply replaces the eigenvalues $\lambda_{n}$ with the new eigenvalues $\phi\left(\lambda_{n}\right)$, while the original and the subordinated semigroup share the same eigenfunctions [compare with (5.3)],

$$
\mathcal{P}_{t}^{\beta, \phi} \varphi_{n}^{\beta}=e^{-\phi\left(\lambda_{n}^{\beta}\right) t} \varphi_{n}^{\beta} \quad \text { and } \quad \mathcal{A}^{\beta, \phi} \varphi_{n}^{\beta}=-\phi\left(\lambda_{n}^{\beta}\right) \varphi_{n}^{\beta} .
$$

Therefore, if the eigenfunction expansion is known for the original semigroup, then it is immediately known for the subordinate semigroup as well. This fact was already pointed out in the original work of Bochner (1949); see equation (11). This allows us to extend analytical tractability of classical diffusion models in finance, such OU, CIR, CEV, etc., to their time-changed (subordinate) counterparts with jumps. This observation has been applied to subordinate OU processes in Li and Linetsky (2013b), to subordinate JDCEV processes in Mendoza-Arriaga, Carr and Linetsky (2010), MendozaArriaga and Linetsky (2013) and to subordinate CIR default intensities in Section 6 of the present paper.

Applying the eigenfunction expansions of semigroups $\left(\mathcal{P}_{t}^{\beta, \phi}\right)_{t \geq 0}$ with $\beta=$ 0,1 , to the pricing of credit-sensitive securities, assuming the payoffs $f_{i}(x) \in$ $L^{2}(I, m)$ in equation (4.1), we immediately obtain the eigenfunction expansion of the value function (4.2),

$$
\begin{aligned}
f\left(t, X_{t}^{\phi}, D_{t}^{\phi} ; T\right)= & e^{-r(T-t)} \sum_{n=1}^{\infty} e^{-\phi\left(\lambda_{n}^{0}\right)(T-t)} f_{n}^{1} \varphi_{n}^{0}\left(X_{t}^{\phi}\right) \\
& +e^{-r(T-t)} \sum_{n=1}^{\infty} e^{-\phi\left(\lambda_{n}^{1}\right)(T-t)} f_{n}^{0-1}\left(1-D_{t}^{\phi}\right) \varphi_{n}^{1}\left(X_{t}^{\phi}\right)
\end{aligned}
$$

with the expansion coefficients

$$
f_{n}^{0-1}=\left(f_{0}-f_{1}, \varphi_{n}^{1}\right) \quad \text { and } \quad f_{n}^{1}=\left(f_{1}, \varphi_{n}^{0}\right) .
$$

We note that the eigenfunction expansion has the following probabilistic interpretation. Due to the eigenfunction property (5.9) each process $\left\{e^{\phi\left(\lambda_{n}^{0}\right) t} \varphi_{n}^{0}\left(X_{t}^{\phi}\right), t \geq 0\right\}$ and $\left\{e^{\phi\left(\lambda_{n}^{1}\right) t}\left(1-D_{t}^{\phi}\right) \varphi_{n}^{1}\left(X_{t}^{\phi}\right), t \geq 0\right\}$ is an $\mathbb{H}^{\phi}$-martingale. Thus, the eigenfunction expansion can be viewed as a martingale expansion. 
In particular, if $f_{0}(x)=1 \in L^{2}(I, m)$ and $f_{1}(x)=0$, we obtain an eigenfunction expansion of the survival probability

$$
\begin{aligned}
& \mathcal{Q}\left(t, X_{t}^{\phi}, D_{t}^{\phi} ; T\right)=\left(1-D_{t}^{\phi}\right) \sum_{n=1}^{\infty} e^{-\phi\left(\lambda_{n}^{1}\right)(T-t)} f_{n} \varphi_{n}^{1}\left(X_{t}^{\phi}\right) \\
& \\
& f_{n}=\left(1, \varphi_{n}^{1}\right) .
\end{aligned}
$$

We note that, due to the existence of the stationary density, $1 \in L^{2}(I, m)$ in the SubCIR model, as well as many other default intensity models, and the survival probability has an eigenfunction expansion. We also remark that in those cases where the speed measure is an infinite measure on $I$, constants are not in $L^{2}(I, m)$. However, it sometimes happens that, while $1 \notin L^{2}(I, m), \mathcal{P}_{t}^{1, \phi} 1 \in L^{2}(I, m)$ for $t>0$ if the semigroup has the property $\mathcal{P}_{t}^{1, \phi} C_{b}(I) \subset L^{2}(I, m)$ for $t>0$.

We conclude this section with an observation that the long-maturity asymptotics of the credit spread of a defaultable zero-coupon bond with zero recovery is simply equal to the principal eigenvalue of the negative of the generator $\mathcal{A}^{1, \phi}$,

$$
\mathcal{S}_{\infty}:=\lim _{T \rightarrow \infty} \mathcal{S}\left(t, X_{t}^{\phi} ; T\right)=\phi\left(\lambda_{0}^{1}\right) .
$$

This immediately follows from the definition of the credit spread (4.4) and the structure of the eigenfunction expansion of the survival probability (5.13).

6. The SubCIR intensity model with two-sided mean-reverting jumps. We now come back to the CIR model of Examples 2.1 and 2.2. We start with the bi-variate process $(X, D)$, where $X$ is a CIR diffusion, and $D$ is a one-point point process with the compensator $A_{t}=\int_{0}^{t}\left(1-D_{s}\right) X_{s} d s$ and time change it with a subordinator. We call the resulting process $\left(X^{\phi}, D^{\phi}\right)$ the subordinate CIR (SubCIR) default intensity model. The default time $\tau$ in this model is the first time default indicator $D^{\phi}$ equals one, and its default intensity process is $\lambda_{t}^{\phi}=\left(1-D_{t}^{\phi}\right) k^{\phi}\left(X_{t}^{\phi}\right)$.

We recall that the CIR process on $I=(0, \infty)$, if the Feller condition is satisfied so zero is inaccessible, or on $I=[0, \infty)$, if the Feller condition is not satisfied so zero is instantaneously reflecting, has a stationary density (2.6). We choose $x_{0}$ in the definition of the speed density (3.26) so that $m(x)=$ $\pi(x)$ [i.e., $\int_{I} m(x) d x=1$ ]. Then for all $\beta \geq 0$ the semigroup $\left(\mathcal{P}_{t}^{\beta}\right)_{t \geq 0}$ defined by (2.3) with the CIR diffusion $X$ and $k(x)=\beta x$ has a symmetric density $p_{m}^{\beta}(t, x, y)$ with respect to the stationary distribution $\pi(y) d y$ given by

$$
p_{m}^{\beta}(t, x, y)=\frac{\rho \Gamma(b) \sqrt{x y}}{\sigma^{2} \sinh (t \rho / 2)}\left(\frac{e^{\rho t / 2}}{a \sqrt{x y}}\right)^{b} I_{b-1}\left(\frac{2 \rho \sqrt{x y}}{\sigma^{2} \sinh (t \rho / 2)}\right)
$$




$$
\times \exp \left\{(x+y)\left(\frac{\kappa \tanh (t \rho / 2)-\rho}{\sigma^{2} \tanh (t \rho / 2)}\right)-\lambda_{0}^{\beta} t\right\},
$$

where $I_{\nu}(x)$ is the modified Bessel function of the first kind and

$$
\lambda_{1}^{\beta}:=\frac{b}{2}(\rho-\kappa) \quad \text { and } \quad \rho:=\rho(\beta)=\sqrt{\kappa^{2}+2 \beta \sigma^{2}},
$$

and $a$ and $b$ are defined in equation (2.6). This explicit solution in terms of the Bessel function is due to the fact that the CIR process can be obtained by a deterministic time change from the squared Bessel process in a similar way as the OU process can be obtained from Brownian motion by a deterministic time change [cf. Proposition 6.3.1.1 and 6.3.2.1 of Jeanblanc, Yor and Chesney (2009), pages 357-358] combined with the absolute continuity relationships for Bessel processes; see Section 6.3 in Jeanblanc, Yor and Chesney (2009), page 340, for more details. For $\beta=1$ this density has appeared in the seminal work of Cox, Ingersoll and Ross (1985) on their interest rate model.

The bi-linear eigenfunction expansion (5.4) for the density $p_{m}^{\beta}(t, x, y)$ can be obtained from the expression (6.1) by applying the Hille-Hardy formula to expand the Bessel function in the bi-linear expansion of generalized Laguerre polynomials $L_{n}^{\nu}(x)$ [cf. Erdelyi (1953), page 189; valid for all $|t|<1$, $\nu>-1, a, b>0]$

$$
\begin{gathered}
\frac{(a b t)^{-\nu / 2}}{1-t} \exp \left\{-\frac{(a+b) t}{1-t}\right\} I_{\nu}\left(\frac{2 \sqrt{a b t}}{1-t}\right) \\
=\sum_{k=0}^{\infty} \frac{t^{k} k !}{\Gamma(k+\nu+1)} L_{k}^{\nu}(a) L_{k}^{\nu}(b) .
\end{gathered}
$$

The application of the Hille-Hardy formula thus yields the eigenfunctions and eigenvalues of the semigroup $\left(\mathcal{P}_{t}^{\beta}\right)_{t \geq 0}$ and its generator $\mathcal{A}^{\beta}$ in the Hilbert space $L^{2}(I, m)$ with $m(d x)=\pi(x) d x$ (the CIR stationary distribution). Due to the appearance of Laguerre polynomials, semigroups of this type are sometimes called Laguerre semigroups in analysis; cf. Nowak and Stempak (2010). The following theorem summarizes the explicit results for eigenvalues and eigenfunctions.

TheOREM 6.1 (CIR eigenfunction expansion). The semigroup $\left(\mathcal{P}_{t}^{\beta}\right)_{t \geq 0}$ is a symmetric trace-class semigroup in $L^{2}(I, m)$ with the eigenvalues and continuous eigenfunctions of the negative of its self-adjoint infinitesimal generator $\mathcal{A}^{\beta}$ given by

$$
\lambda_{n}^{\beta}=(n-1) \rho+\frac{b}{2}(\rho-\kappa),
$$




$$
\begin{aligned}
\varphi_{n}^{\beta}(x) & =\mathcal{N}_{n}^{\beta} e^{((\kappa-\rho) x) / \sigma^{2}} L_{n-1}^{b-1}\left(\frac{2 x \rho}{\sigma^{2}}\right), \\
\mathcal{N}_{n}^{\beta} & =\sqrt{\frac{(n-1) !}{(b)_{n-1}}}\left(\frac{\rho}{\kappa}\right)^{b / 2}, \quad n=1,2, \ldots,
\end{aligned}
$$

where $(a)_{n}=\Gamma(a+n) / \Gamma(a)=a(a+1) \cdots(a+n-1)$ is the Pochhammer symbol. Moreover, on each compact interval $K \subset I$ there exists a constant $C_{K}$ independent of $n$ such that

$$
\left|\varphi_{n}^{\beta}(x)\right| \leq C_{K} n^{-1 / 4}
$$

or all $n \geq 1$.

Proof. The bi-linear expansion for the density of the form (5.4) with $\varphi_{n}^{\beta}(x)$ and $\lambda_{n}^{\beta}$ given by equations (6.3) and (6.4) is directly obtained by applying the Hille-Hardy formula (6.2) to the right-hand side of equation (6.1). It is then easy to directly verify from the properties of Laguerre polynomials that $\varphi_{n}^{\beta}(x)$ are eigenfunctions of the operator

$$
\mathcal{A}^{\beta} f=\frac{1}{2} \sigma^{2} x f^{\prime \prime}(x)+\kappa(\theta-x) f^{\prime}(x)-\beta x f(x)
$$

with eigenvalues $\lambda_{n}^{\beta}$ satisfying the boundary condition at zero $\lim _{x \downarrow 0}\left(\varphi^{\beta}(x)\right)^{\prime} /$ $s(x)=0$, where $s(x)$ is the scale density defined in equation (3.26). The eigenfunctions are normalized with respect to the inner product with $m(d x)=$ $\pi(x) d x,\left(\varphi_{n}^{\beta}, \varphi_{m}^{\beta}\right)=\delta_{n, m}$. The trace class condition (5.1) is verified due to the linear growth of eigenvalues. The bound for the eigenfunctions is obtained from the estimate in equation (27a) of Nikiforov and Uvarov (1988), page 54 .

This CIR eigenfunction expansion has been applied in finance in Davydov and Linetsky (2003) and Gorovoi and Linetsky (2004) [we note that our normalization factor $\mathcal{N}_{n}$ in the expression for eigenfunctions differs from Davydov and Linetsky (2003), Proposition 9, due to different normalization of the speed measure; here we normalize the speed measure so it integrates to one and thus coincides with the stationary distribution].

For any $f \in L^{2}(I, m)$ the computation of $\mathcal{P}_{t}^{\beta} f(x)$ reduces to computing the expansion coefficients. In particular, consider the discounted CIR characteristic function known in closed form due to the fact that the CIR diffusion is a CBI/affine process; cf. Cox, Ingersoll and Ross (1985), Duffie and Garleanu (2001), Appendix A. For any complex $z$ with $\Re z \geq 0$,

$$
\begin{aligned}
\Psi_{t}(x, \beta, z) & :=\mathbb{E}_{x}\left[e^{-\beta \int_{0}^{t} X_{u} d u} e^{-z X_{t}}\right] \\
& =A(t, \beta, z) \exp \{-B(t, \beta, z) x\},
\end{aligned}
$$


where

$$
\begin{aligned}
A(t, \beta, z) & :=\left(\frac{2 \rho e^{(\kappa+\rho) t / 2}}{2 \rho+\left(\rho+\kappa+z \sigma^{2}\right)\left(e^{\rho t}-1\right)}\right)^{b}, \\
B(t, \beta, z) & :=\frac{2 \beta\left(e^{\rho t}-1\right)+z(\rho-\kappa) e^{\rho t}+z(\rho+\kappa)}{2 \rho+\left(\rho+\kappa+z \sigma^{2}\right)\left(e^{\rho t}-1\right)} .
\end{aligned}
$$

We have the following eigenfunction expansion of the characteristic function.

Proposition 6.1. The characteristic function has the eigenfunction expansion (5.8) with the coefficients given by

$$
f_{1}^{\beta}(z)=1, \quad f_{n}^{\beta}(z)=\frac{1}{\mathcal{N}_{n}^{\beta}}\left(\frac{\kappa-\rho+\sigma^{2} z}{\kappa+\rho+\sigma^{2} z}\right)^{n-1}\left(\frac{2 \rho}{\kappa+\rho+\sigma^{2} z}\right)^{b},
$$

$$
n=2, \ldots
$$

Proof. Obtained immediately from the identity for the generating function of the generalized Laguerre polynomials (valid for all complex $|y|<1$ and $a>-1)$,

$$
\sum_{k=0}^{\infty} y^{k} L_{k}^{a}(x)=(1-y)^{-1-a} \exp ((y x) /(y-1)) .
$$

Alternatively, the integrals in $f_{n}^{\beta}(z)=\left(e^{-z}, \varphi_{n}^{\beta}\right)$ can be explicitly calculated due to the integral identity for Laguerre polynomials in equation (2.19.3.3), Prudnikov, Brychkov and Marichev (1986), page 462.

We note that if one is only interested in the CIR characteristic function, the affine closed-form expression (6.5) is certainly simpler than the eigenfunction expansion. However, while the affine expression (6.5) does not generalize to the SubCIR model, the eigenfunction expansion generalizes immediately, yielding

$$
\Psi_{t}^{\phi}(x, \beta, z):=\left(\mathcal{P}_{t}^{\beta, \phi} e^{-z \cdot}\right)(x)=\sum_{n=1}^{\infty} e^{-\phi\left(\lambda_{n}^{\beta}\right) t} f_{n}^{\beta}(z) \varphi_{n}^{\beta}(x)
$$

with the same eigenfunctions and expansion coefficients (6.6) but with new eigenvalues $\phi\left(\lambda_{n}^{\beta}\right)$, where $\phi$ is the Laplace exponent of the subordinator.

In particular, the eigenfunction expansion for the survival probability (4.3) in the SubCIR default intensity model is then immediately obtained

$$
\begin{aligned}
\mathcal{Q}\left(t, X_{t}^{\phi}, D_{t}^{\phi} ; T\right) & =\left(1-D_{t}^{\phi}\right) P_{T-t}^{1, \phi}\left(X_{t}^{\phi}, I\right) \\
& =\left(1-D_{t}^{\phi}\right) \Psi_{T-t}^{\phi}\left(X_{t}^{\phi}, 1,0\right)
\end{aligned}
$$




$$
=\left(1-D_{t}^{\phi}\right) \sum_{n=1}^{\infty} e^{-\phi\left(\lambda_{n}^{1}\right)(T-t)} f_{n}^{1}(0) \varphi_{n}^{1}\left(X_{t}^{\phi}\right)
$$

by setting $z=0$ in the expansion for the characteristic function. The pricing of zero-coupon bonds with constant recovery (4.5) is then immediate. The pricing of other credit-sensitive securities in the SubCIR default intensity model then reduces to computing the corresponding expansion coefficients in equations (5.10)-(5.11). In particular, the pricing and calibration of credit default swaptions is considered in Mendoza-Arriaga (2012).

We also remark that the same eigenfunction expansion yields the pricing of default-free zero-coupon bonds in the SubCIR interest rate model of Remark 4.1,

$$
\begin{aligned}
P\left(Z_{t}^{\phi}, t ; T\right) & =P_{T-t}^{1, \phi}\left(Z_{t}^{\phi}, I\right)=\Psi_{T-t}^{\phi}\left(X_{t}^{\phi}, 1,0\right) \\
& =\sum_{n=1}^{\infty} e^{-\phi\left(\lambda_{n}^{1}\right)(T-t)} f_{n}^{1}(0) \varphi_{n}^{1}\left(Z_{t}^{\phi}\right) .
\end{aligned}
$$

We now present a numerical illustration of the qualitative properties of the SubCIR default intensity model. We start with a CIR process $X$ with $\kappa=1, \theta=0.1$ and $\sigma=0.25$. The SubCIR process $X^{\phi}$ is constructed by subordinating $X$ with an inverse Gaussian subordinator $\left(\mathcal{T}_{t}\right)_{t \geq 0}$ with the Lévy measure (3.1) with parameters $\alpha=0.5, \eta=1$ and $C=0.5$, and zero drift $\gamma=0$. Since the subordinator is driftless, $X^{\phi}$ is a pure jump process in our example. Figure 1(a) shows simulation of a typical sample path of the CIR process $X$ and the SubCIR process $X^{\phi}$ with these parameters. While the CIR process diffuses around its long-run level $\theta$ with volatility $\sigma$, while being pulled back toward it by the mean-reverting drift at the rate

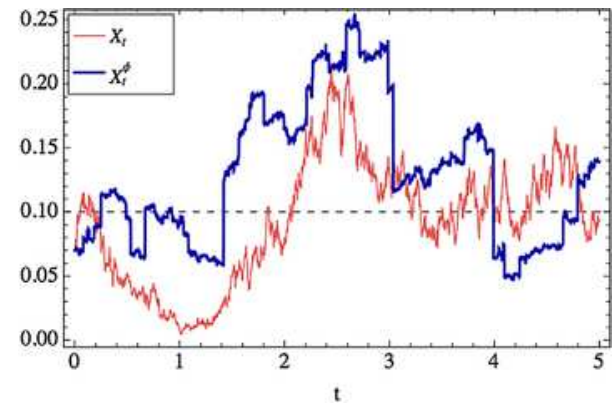

(a) Sample paths

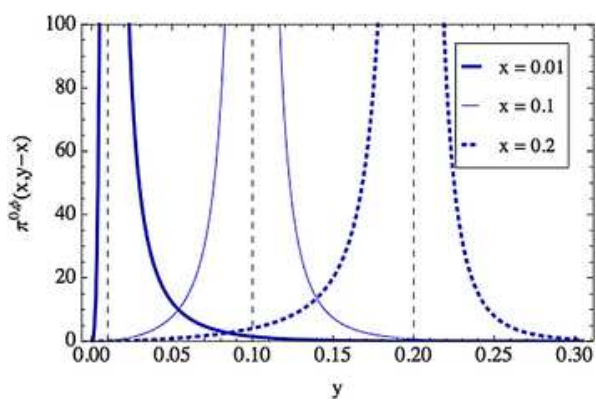

(b) Lévy densities

FIG. 1. (a) Sample paths of a CIR process $\left(X_{t}\right)_{t \geq 0}$ and the SubCIR process $\left(X_{t}^{\phi}\right)_{t \geq 0}$. The horizontal line (dashed) corresponds to the long run mean level $\theta=0.1$. Figure (b) contains three jump densities $\pi^{0, \phi}(x, y-x)$ corresponding to the initial states $x=0.01$, $x=\theta=0.1$ and $x=0.2$, which are indicated by the vertical lines (dashed). 
$\kappa$, the SubCIR process is a pure jump process with state-dependent meanreverting Lévy measure. The mean-reverting nature of jumps is evident in the sample path plot (a), as well as in the plot (b) of the Lévy density $\pi^{0, \phi}(x, y-x)=m(y) \int_{(0, \infty)} p_{m}^{0}(s, x, y) \nu(d s)$ plotted as a function of $y$ for three fixed values $x$. This plot shows three Lévy densities of jumps from the three initial states $x=0.01, x=\theta=0.1$ and $x=0.2$. Here $x$ is the pre-jump state, and $y$ is the post-jump state, so that the jump size is $y-x$. When $x=\theta=0.1$, that is, jumping from the long-run mean, the Lévy density looks nearly symmetrical. In contrast, the Lévy density of jumps starting from the state $x=0.01<0.1$ significantly below the long-run mean is highly skewed to the right, as the process tends to jump back up toward its long run mean at 0.1 from this low value of 0.01 . On the other hand, the Lévy density of jumps starting from the state $x=0.2>0.1$ significantly above the long-run mean is highly skewed to the left, as the process tends to jump back down toward its long run mean at 0.1 from this high value of 0.2 . Either way, the process stays nonnegative. This is in sharp contrast with the behavior of affine jump-diffusion/CBI-processes that can only jump up and cannot jump down to ensure that the process stays nonnegative. In the framework of subordinate diffusions, the nonnegativity of SubCIR process is immediate, as the subordinate process and the original process share the same state space.

From the expression for $k^{\phi}(x)$ arising from Theorem 3.2,

$$
k^{\phi}(x)=\gamma \beta x+\int_{(0, \infty)}(1-A(s, \beta, 0) \exp \{-B(s, \beta, 0) x\}) \nu(d s),
$$

where we substituted the closed-form expression for the survival probability of the CIR process, it is clear that the default intensity is no longer affine as in the SubCIR model. Figure 2 illustrates a sample path of the default intensity process $\lambda_{t}^{\phi}=\left(1-D_{t}^{\phi}\right) k^{\phi}\left(X_{t}^{\phi}\right)$, along with a sample path of the pure jump process $X^{\phi}$.

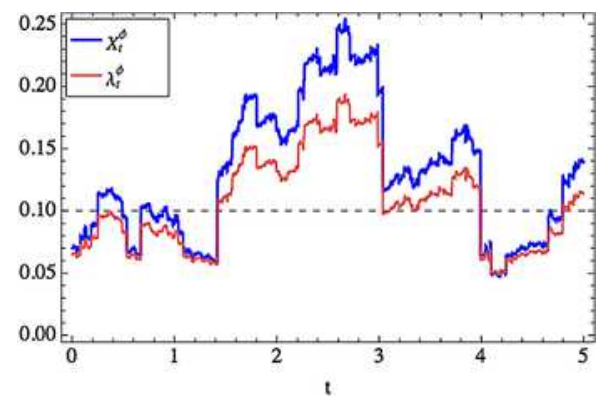

FIG. 2. Default intensity $\lambda_{t}^{\phi}=\left(1-D_{t}^{\phi}\right) k^{\phi}\left(X_{t}^{\phi}\right)$. This figure illustrates the sample path of the default intensity process $\left(\lambda_{t}^{\phi}\right)_{t \geq 0}$ induced by the SubCIR process $\left(X_{t}^{\phi}\right)_{t \geq 0}$, which is also depicted. The horizontal line (dashed) corresponds to the long run mean level $\theta=0.1$. 


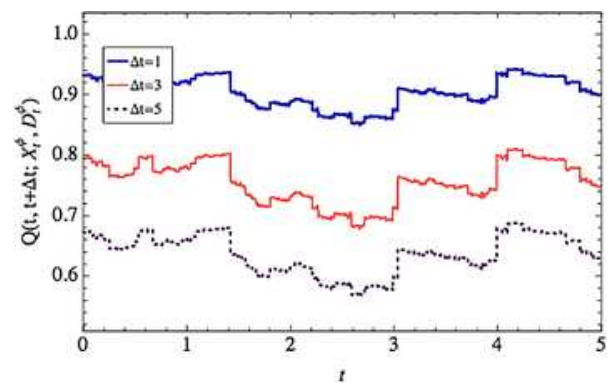

(a) Survival probability

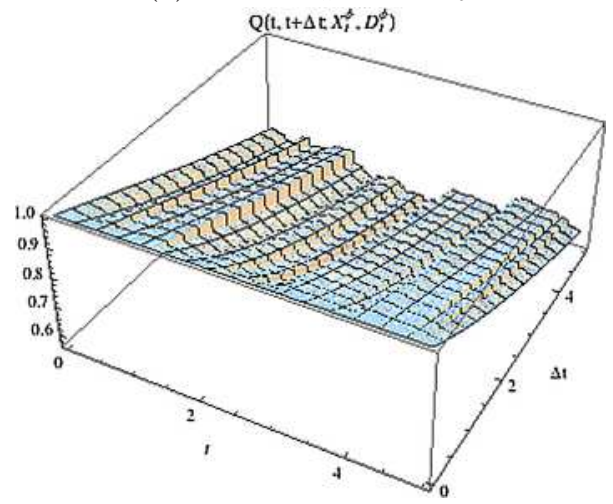

(c) Survival probability

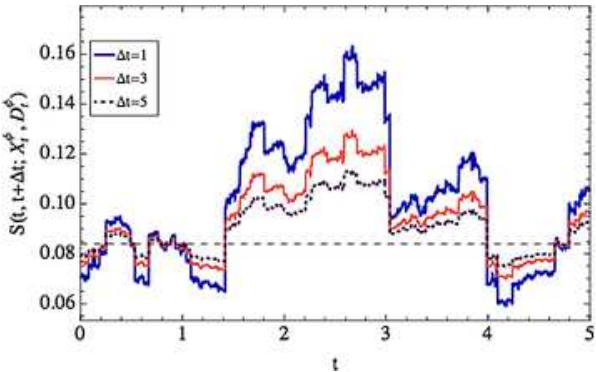

(b) Credit spreads

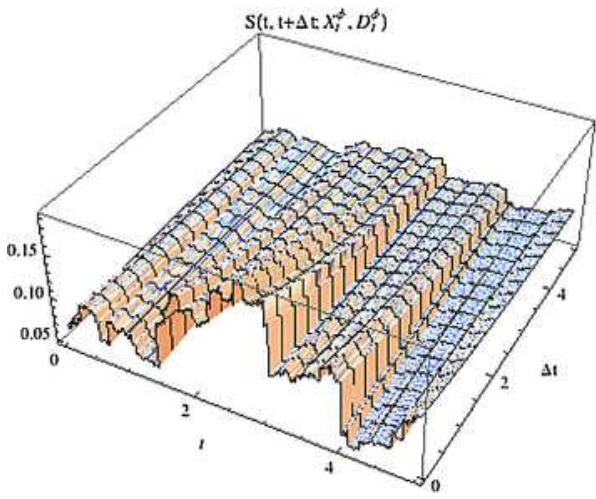

(d) Credit spreads

Fig. 3. Survival Probabilities and Credit Spreads sample paths.

Finally, Figure 3 shows sample paths of the survival probabilities (4.3) and defaultable credit spreads on zero-coupon bonds (4.4) over a five-year period simulated under this SubCIR default intensity specification. Figure 3(a) and $3(\mathrm{~b})$ show sample paths of the survival probabilities for one, three and five years, that is, $\mathcal{Q}\left(t, t+\Delta t ; X_{t}^{\phi}, D_{t}^{\phi}\right)$ with $\Delta t=1,3,5$ years, and one- threeand five-year credit spreads, $\mathcal{S}\left(t, X_{t}^{\phi}, D_{t}^{\phi}, t+\Delta t\right)$, respectively. The dashed horizontal line in (b) corresponds to the asymptotic credit spread $\mathcal{S}_{\infty}=0.084$ equal to the principal eigenvalue $\phi\left(\lambda_{1}^{1}\right)$ of the semigroup $\mathcal{P}^{1, \phi}$. Figure $3(\mathrm{c})$ and $3(\mathrm{~d})$ show sample paths over five years of the evolution of the term structure of survival probabilities $\mathcal{Q}\left(t, X_{t}^{\phi}, D_{t}^{\phi} ; t+\Delta t\right)$ and credit spreads $\mathcal{S}\left(t, X_{t}^{\phi}, D_{t}^{\phi} ; t+\Delta t\right)$, respectively. Since the SubCIR state variable $X^{\phi}$ is a jump process, prices of credit-sensitive securities, such as bond prices, as well as credit spreads, are also jump processes in this model.

7. Conclusion. The present paper introduces a jump-diffusion extension of the classical diffusion default intensity model by means of subordination in the sense of Bochner. We start with the bi-variate process of the diffusion 
state variable and default indicator $(X, D)$ in the diffusion intensity framework and time change it with a Lévy subordinator $\mathcal{T}$. We characterize the resulting time changed process $\left(X_{t}^{\phi}, D_{t}^{\phi}\right)=\left(X\left(\mathcal{T}_{t}\right), D\left(\mathcal{T}_{t}\right)\right)$ as a Markovian Itô semimartingale and, in particular, show from the Doob-Meyer decomposition of $D^{\phi}$ that the default time in the time-changed model has a jumpdiffusion or pure jump intensity. When $X$ is a CIR diffusion with meanreverting drift, the default intensity of the subordinate model (SubCIR) is a nonnegative jump-diffusion or pure jump process with two-sided meanreverting jumps that stays nonnegative. The SubCIR default intensity model is fully analytically tractable by means of the explicitly computed eigenfunction expansion of the relevant semigroups. This yields explicit closed-form pricing of credit-sensitive securities.

\section{REFERENCES}

Ahn, D.-H. and GaO, B. (1999). A parametric nonlinear model of term structure dynamics. The Review of Financial Studies 12 721-762.

Andreasen, J. (2001). Credit explosives. Bank of America Fixed Income Research Working Paper.

Barlow, M. T. (2002). A diffusion model for electricity prices. Math. Finance 12 287-298.

Barndorff-Nielsen, O. E. (1998). Processes of normal inverse Gaussian type. Finance Stoch. 2 41-68. MR1804664

Beaglehole, D. and Tenney, M. (1992). Corrections and additions to "A nonlinear equilibrium model of the term structure of interest rates." Math. Finance 32 345-353.

Bertoin, J. (1996). Lévy Processes. Cambridge Tracts in Mathematics 121. Cambridge Univ. Press, Cambridge. MR1406564

Bertoin, J. (1999). Lectures on Probability Theory and Statistics. Lecture Notes in Math. 1717. Springer, Berlin. MR1746299

Bielecki, T. R., Jeanblanc, M. and Rutkowski, M. (2011). Hedging of a credit default swaption in the CIR default intensity model. Finance Stoch. 15 541-572. MR2833099

Bielecki, T. R. and Rutkowski, M. (2004). Credit Risk: Modeling, Valuation and Hedging. Springer, Berlin.

Bielecki, T. R., Crépey, S., Jeanblanc, M. and Rutkowski, M. (2008). Defaultable options in a Markovian intensity model of credit risk. Math. Finance 18 493-518. MR2454669

Bielecki, T. R., Cousin, A., Crépey, S. and Herbertsson, A. (2013). Dynamic modeling of portfolio credit risk with common shocks. J. Optim. Theory Appl. To appear.

Bielecki, T. R., Crépey, S., Jeanblanc, M. and Zargari, B. (2012). Valuation and hedging of CDS counterparty exposure in a Markov copula model. Int. J. Theor. Appl. Finance 15 1250004, 39. MR2902965

Bochner, S. (1949). Diffusion equation and stochastic processes. Proc. Natl. Acad. Sci. USA 35 368-370. MR0030151

Borodin, A. N. and Salminen, P. (2002). Handbook of Brownian Motion-Facts and Formulae, 2nd ed. Birkhäuser, Basel. MR1912205

Boyarchenko, N. and Levendorskil̆, S. (2007). The eigenfunction expansion method in multi-factor quadratic term structure models. Math. Finance 17 503-539. MR2352904

Brigo, D. and Alfonsi, A. (2005). Credit default swap calibration and derivatives pricing with the SSRD stochastic intensity model. Finance Stoch. 9 29-42. MR2210926 
Brigo, D. and El-BAChir, N. (2006). Credit derivatives pricing with a smile-extended jump stochastic intensity model. Working paper.

Brigo, D. and El-BACHIR, N. (2010). An exact formula for default swaptions' pricing in the SSRJD stochastic intensity model. Math. Finance 20 365-382. MR2667895

CArr, P. and Linetsky, V. (2006). A jump to default extended CEV model: An application of Bessel processes. Finance Stoch. 10 303-330. MR2244347

ÇINLAR, E. and JACOD, J. (1981a). Representation of semimartingale Markov processes in terms of Wiener processes and Poisson random measures. In Seminar on Stochastic Processes, 1981 (Evanston, Ill., 1981) (E. Cinlar, K. L. Chung and R. K. Getoor, eds.). Progr. Prob. Statist. 1 159-242. Birkhäuser, Boston, MA. MR0647786

ÇINLAR, E. and JACOD, J. (1981b). Semimartingales defined on Markov processes. In Stochastic Differential Systems (Visegrád, 1980) (M. Arató, D. Vermes and A. V. Balakrishnan, eds.). Lecture Notes in Control and Information Sci. 36 1324. Springer, Berlin. MR0653642

Çinlar, E., Jacod, J., Protter, P. and Sharpe, M. J. (1980). Semimartingales and Markov processes. Z. Wahrsch. Verw. Gebiete 54 161-219. MR0597337

Chen, Z.-Q. and Fukushima, M. (2011). Symmetric Markov Processes, Time Change, and Boundary Theory. Princeton Univ. Press, Princeton, NJ. MR2849840

Cox, J. C., Ingersoll, J. E. JR. and Ross, S. A. (1985). A theory of the term structure of interest rates. Econometrica 53 385-407. MR0785475

Cuchiero, C., Filipović, D., Mayerhofer, E. and Teichmann, J. (2011a). Affine processes on positive semidefinite matrices. Ann. Appl. Probab. 21 397-463. MR2807963

Cuchiero, C., Keller-Ressel, M., Mayerhofer, E. and Teichmann, J. (2011b). Affine processes on symmetric cones. Unpublished manuscript.

Davies, E. B. (2007). Linear Operators and Their Spectra. Cambridge Studies in Advanced Mathematics 106. Cambridge Univ. Press, Cambridge. MR2359869

Davydov, D. and Linetsky, V. (2003). Pricing options on scalar diffusions: An eigenfunction expansion approach. Oper. Res. 51 185-209. MR1964993

Duffie, D., Filipović, D. and Schachermayer, W. (2003). Affine processes and applications in finance. Ann. Appl. Probab. 13 984-1053. MR1994043

Duffie, D. and Garleanu, N. (2001). Risk and valuation of collateralized debt obligations. Financial Analysts Journal 57 41-59.

Duffie, D. and KAN, R. (1996). A yield-factor model of interest rates. Math. Finance 6 379-406.

Duffie, D., Pan, J. and Singleton, K. (2000). Transform analysis and asset pricing for affine jump-diffusions. Econometrica 68 1343-1376. MR1793362

Duffie, D. and Singleton, K. J. (1999). Modeling term structures of defaultable bonds. The Review of Financial Studies 12 687-720.

Duffie, D. and Singleton, K. J. (2003). Credit Risk: Pricing, Measurement, and Management. Princeton Univ. Press, Princeton, NJ.

Elkamhi, R., Jacobs, K., Langlois, H. and Ornthanalai, C. (2012). Accounting information releases and CDS spreads. Working paper.

Erdelyi, A. (1953). Higher Transcendental Functions II. McGraw-Hill, New York.

Ethier, S. N. and Kurtz, T. G. (1986). Markov Processes: Characterization and Convergence. Wiley, New York. MR0838085

Feller, W. (1951). Two singular diffusion problems. Ann. of Math. (2) 54 173-182. MR0054814

Filipović, D. (2001). A general characterization of one factor affine term structure models. Finance Stoch. 5 389-412. MR1850789 
Fukushima, M., Oshima, Y. and Takeda, M. (2011). Dirichlet Forms and Symmetric Markov Processes, extended ed. de Gruyter Studies in Mathematics 19. de Gruyter, Berlin. MR2778606

Geman, H. and Roncoroni, A. (2006). Understanding the fine structure of electricity prices. Journal of Business 79 1225-1261.

GöInG-JAeschke, A. and Yor, M. (2003). A survey and some generalizations of Bessel processes. Bernoulli 9 313-349. MR1997032

Gorovoi, V. and Linetsky, V. (2004). Black's model of interest rates as options, eigenfunction expansions and Japanese interest rates. Math. Finance 14 49-78. MR2030835

Itô, K. and McKean, H. P. (1974). Diffusion Processes and Their Sample Paths, corrected 2nd ed. Springer, Berlin. MR0345224

JACOD, J. (1979). Calcul Stochastique et Problèmes de Martingales. Lecture Notes in Math. 714. Springer, Berlin. MR0542115

Jacod, J. and Protter, P. (2011). Discretization of Processes. Springer, Berlin.

Jacod, J. and Shiryaev, A. N. (2002). Limit Theorems for Stochastic Processes, 2nd. ed. Comprenhensive Studies in Mathematics 288. Springer, Berlin.

JAMShidiAn, F. (1996). Bond, futures and option evaluation in the quadratic interest rate model. Appl. Math. Finance 3 93-115.

Janson, S., M'Baye, S. and Protter, P. (2011). Absolutely continuous compensators. Int. J. Theor. Appl. Finance 14 335-351. MR2804101

Jarrow, R. A., Lando, D. and Turnbull, S. M. (1997). A Markov model for the term structure of credit risk spreads. The Review of Financial Studies 10 481-523.

Jeanblanc, M., Yor, M. and Chesney, M. (2009). Mathematical Methods for Financial Markets. Springer, London. MR2568861

KaWAZU, K. and Watanabe, S. (1971). Branching processes with immigration and related limit theorems. Theory Probab. Appl. 16 36-54.

Keller-Ressel, M., Schachermayer, W. and Teichmann, J. (2011). Affine processes are regular. Probab. Theory Related Fields 151 591-611. MR2851694

KITA, A. (2012). CDS spreads explained with credit spread volatility and jump risk of individual firms. Working paper.

LEwIS, A. L. (1994). Three expansion regimes for interest rate term structure models. Analytic Investment Management. Available at http://optioncity.net/.

Li, L. and Linetsky, V. (2013a). Optimal stopping and early exercise: An eigenfunction expansion approach. Oper. Res. 61 625-644.

Li, L. and Linetsky, V. (2013b). Time-changed Ornstein-Uhlenbeck processes and their applications in commodity derivative models. Math. Finance. To appear.

Lim, D., Li, L. and Linetsky, V. (2012). Evaluating callable and putable bonds: An eigenfunction expansion approach. J. Econom. Dynam. Control 36 1888-1908. MR2982959

Linetsky, V. (2004). The spectral decomposition of the option value. Int. J. Theor. Appl. Finance 7 337-384. MR2064020

Linetsky, V. (2006). Pricing equity derivatives subject to bankruptcy. Math. Finance 16 255-282. MR2212266

LinETSKY, V. (2008). Spectral methods in derivatives pricing. In Handbooks in Operations Research and Management Science: Financial Engineering 15 223-300. Elsevier/NorthHolland, Amsterdam.

Lorig, M., Lozano-Carbassé, O. and Mendoza-Arriaga, R. (2013). Variance swaps on defaultable assets and market implied time-changes. Unpublished manuscript.

Madan, D. B., CARr, P. and Chang, E. C. (1998). The variance gamma process and option pricing. European Finance Review 2 79-105. 
McKean, H. P. JR. (1956). Elementary solutions for certain parabolic partial differential equations. Trans. Amer. Math. Soc. 82 519-548. MR0087012

Mendoza-Arriaga, R. (2012). Credit default swap options under the subordinate diffusion framework. Working paper.

Mendoza-Arriaga, R., Carr, P. and Linetsky, V. (2010). Time-changed Markov processes in unified credit-equity modeling. Math. Finance 20 527-569. MR2731407

Mendoza-Arriaga, R. and Linetsky, V. (2013). Multivariate subordination of Markov processes with financial applications. Math. Finance. To appear.

Meyer-Brandis, T. and Tankov, P. (2008). Multi-factor jump-diffusion models of electricity prices. Int. J. Theor. Appl. Finance 11 503-528.

Nikiforov, A. F. and Uvarov, V. B. (1988). Special Functions of Mathematical Physics. Birkhäuser, Basel. MR0922041

Nowak, A. and Stempak, K. (2010). On $L^{p}$-contractivity of Laguerre semigroups. Working paper.

Phillips, R. S. (1952). On the generation of semigroups of linear operators. Pacific J. Math. 2 343-369. MR0050797

Pitman, J. and Yor, M. (1982). A decomposition of Bessel bridges. Z. Wahrsch. Verw. Gebiete 59 425-457. MR0656509

Prudnikov, A. P., Brychkov, Y. A. and Marichev, O. I. (1986). Integrals Series: Special Functions. Integrals and Series II. Gordon \& Breach, New York.

Reed, M. and Simon, B. (1980). Methods of Modern Mathematical Physics I: Functional Analysis, revised ed. Academic Press, San Diego, CA. MR0751959

Revuz, D. and Yor, M. (1999). Continuous Martingales and Brownian Motion, 3rd ed. Grundlehren der Mathematischen Wissenschaften 293. Springer, Berlin. MR1725357

Sato, K.-I. (1999). Lévy Processes and Infinitely Divisible Distributions. Cambridge Studies in Advanced Mathematics 68. Cambridge Univ. Press, Cambridge. MR1739520

Schilling, R. L., Song, R. and Vondraček, Z. (2010). Bernstein Functions: Theory and Applications. de Gruyter Studies in Mathematics 37. de Gruyter, Berlin. MR2598208

ZhANG, B. Y., ZhоU, H. and ZHU, H. (2009). Explaining credit default swap spreads with the equity volatility and jump risks of individual firms. Review of Financial Studies 22 5099-5131.

IROM

McCombs School of Business

University of TeXas at Austin

CBA 5.202, B6500

Austin, TeXas 78712

USA

E-MAIL: rafael.mendoza-arriaga@mccombs.utexas.edu
IEMS

McCormick School of Engineering And Applied Sciences NORTHWESTERN UNIVERSITY 2145 SHERIDAN ROAD EVANSTON, ILLINOIS 60208

USA

E-MAIL: linetsky@iems.northwestern.edu 